\title{
Some open problems in elasticity
}

\author{
J. M. Ball
}

Dedicated to Jerry Marsden on the occasion of his 60th birthday

\begin{abstract}
Some outstanding open problems of nonlinear elasticity are described. The problems range from questions of existence, uniqueness, regularity and stability of solutions in statics and dynamics to issues such as the modelling of fracture and self-contact, the status of elasticity with respect to atomistic models, the understanding of microstructure induced by phase transformations, and the passage from three-dimensional elasticity to models of rods and shells. Refinements are presented of the author's earlier work Ball [1984a] on showing that local minimizers of the elastic energy satisfy certain weak forms of the equilibrium equations.
\end{abstract}

\section{Contents}

1 Some open problems in elasticity 1

1 Introduction. . . . . . . . . . . . . 2

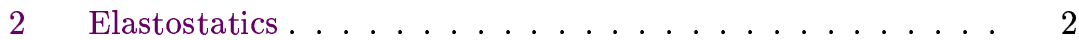

2.1 The stored-energy function and equilibrium solutions 3

2.2 Existence of equilibrium solutions . . . . . . . 5

2.3 Regularity and the classification of singularities . . . 9

2.4 Satisfaction of the Euler-Lagrange equation and uniform positivity of the Jacobian. . . . . . . . . 13

2.5 Regularity and self-contact .......... 19

2.6 Uniqueness of solutions ............ 20

2.7 Structure of the solution set . . . . . . . . . . 21

2.8 Energy minimization and fracture. ....... 24

3 Dynamics ..................... 25

3.1 Continuum thermomechanics ......... 25

3.2 Existence of solutions . . . . . . . . . 27

3.3 The relation between statics and dynamics . . . . 31

4 Multiscale problems ..................... 34

4.1 From atomic to continuum ......... 34

4.2 From microscales to macroscales ......... 36

Version Dec 16, 2001 ....... Edited by wgm : Typeset on 26 January $2002-9$ h38 
4.3 From three-dimensional elasticity to theories of rods and shells ................. 41

\section{Introduction.}

In this paper I highlight some outstanding open problems in nonlinear (sometimes called finite) elasticity theory. While many of these will be well known to experts on analytic aspects of elasticity, I hope that the compilation will be of use both to those new to the field and to researchers in solid mechanics having different perspectives. Of course the selection of problems is a personal one, and indeed represents a list of those problems I would like most to be able to solve but cannot. In particular it concentrates on general open problems, or ones that illustrate general difficulties, rather than those related to very specific experimental situations, which is not to imply that the latter are not important or instructive. I have not included any open problems connected with the numerical computation of solutions, since I recently discussed some of these in Ball [2001].

The only new results of the paper are in connection with the problem of showing that local minimizers of the total elastic energy satisfy the weak form of the equilibrium equations. As I pointed out in Ball [1984a], there are hypotheses under which some forms of the equilibrium equations can be proved to hold, and in Section $2.4 \mathrm{I}$ take the opportunity to present some refinements of this old work.

The paper is essentially self-contained, and can be read by those having no knowledge of elasticity theory. For those seeking further background on the subject I have written a short introduction Ball [1996] to some of the issues, intended for research students, which I hope is a quick and easy read. For more serious study in the spirit of this paper, the reader is referred to the books of Antman [1995], Ciarlet [1988, 1997, 2000], Marsden and Hughes [1983] and Šilhavý [1997]. Other excellent but older books and survey articles are Antman [1983], Ericksen [1977b], Gurtin [1981] and Truesdell and Noll [1965]. Valuable additional perspectives can be found in the books of Green and Zerna [1968], Green and Adkins [1970], and Ogden [1984].

It is an honour to dedicate this article to Jerry Marsden, both as a friend and in recognition of his important contributions to elasticity, and thus to help celebrate his many talents as a mathematician, thinker and writer.

\section{Elastostatics}

Version Dec 16, $2001 \ldots . .$. Edited by wgm : Typeset on 26 January $2002-9$ h38 


\subsection{The stored-energy function and equilibrium solutions}

Consider an elastic body which in a reference configuration occupies the bounded domain $\Omega \subset \mathbf{R}^{3}$. We suppose that $\Omega$ has a Lipschitz boundary $\partial \Omega=\partial \Omega_{1} \cup \partial \Omega_{2} \cup N$, where $\partial \Omega_{1}, \partial \Omega_{2}$ are disjoint relatively open subsets of $\partial \Omega$ and $N$ has two-dimensional Hausdorff measure $\mathcal{H}^{2}(N)=0$ (i.e., $N$ has zero area). Deformations of the body are described by mappings

$$
\mathbf{y}: \Omega \rightarrow \mathbf{R}^{3}
$$

where $\mathbf{y}(\mathbf{x})=\left(y_{1}(\mathbf{x}), y_{2}(\mathbf{x}), y_{3}(\mathbf{x})\right)$ denotes the deformed position of the material point $\mathbf{x}=\left(x_{1}, x_{2}, x_{3}\right)$. We assume that $\mathbf{y}$ belongs to the Sobolev space $W^{1,1}\left(\Omega ; \mathbf{R}^{3}\right)$, so that in particular the deformation gradient $D \mathbf{y}(\mathbf{x})$ is well defined for a.e. $\mathbf{x} \in \Omega$. For each such $\mathbf{x}$ we can identify $D \mathbf{y}(\mathbf{x})$ with the $3 \times 3$ matrix $\left(\partial y_{i} / \partial x_{j}\right)$.

We require the deformation $\mathbf{y}$ to satisfy the boundary condition

$$
\left.\mathbf{y}\right|_{\partial \Omega_{1}}=\overline{\mathbf{y}}(\cdot)
$$

where $\overline{\mathbf{y}}: \partial \Omega_{1} \rightarrow \mathbf{R}^{3}$ is a given boundary displacement.

We suppose for simplicity that the body is homogeneous, i.e., the material response is the same at each point. In this case the total elastic energy corresponding to the deformation $\mathbf{y}$ is given by

$$
I(\mathbf{y})=\int_{\Omega} W(D \mathbf{y}(\mathbf{x})) d \mathbf{x},
$$

where $W=W(\mathbf{A})$ is the stored-energy function of the material. We suppose that $W: M_{+}^{3 \times 3} \rightarrow \mathbf{R}$ is $C^{1}$ and bounded below, so that without loss of generality $W \geq 0$. (Here and below, $M^{m \times n}$ denotes the space of real $m \times n$ matrices, and $M_{+}^{n \times n}$ denotes the space of those $\mathbf{A} \in M^{n \times n}$ with $\operatorname{det} \mathbf{A}>0$.) The Piola-Kirchhoff stress tensor is given by

$$
\mathbf{T}_{R}(\mathbf{A})=D_{\mathbf{A}} W(\mathbf{A})
$$

By formally computing

$$
\left.\frac{d}{d \tau} I(\mathbf{y}+\tau \varphi)\right|_{\tau=0}=0,
$$

we obtain the weak form of the Euler-Lagrange equation for $I$, that is

$$
\int_{\Omega} D_{\mathbf{A}} W(D \mathbf{y}) \cdot D \varphi d \mathbf{x}=0
$$

for all smooth $\varphi$ with $\left.\varphi\right|_{\partial \Omega_{1}}=0$. This can be shown (cf. Antman and Osborn [1979]) to be equivalent to the balance of forces on arbitary subbodies. 
If $\mathbf{y}, \partial \Omega_{1}$ and $\partial \Omega_{2}$ are sufficiently regular then (2.4) is equivalent to the pointwise form of the equilibrium equations

$$
\operatorname{div} D_{\mathbf{A}} W(D \mathbf{y})=0 \quad \text { in } \Omega,
$$

together with the natural boundary condition of zero applied traction

$$
D_{\mathbf{A}} W(D \mathbf{y}) \mathbf{n}=0 \quad \text { on } \partial \Omega_{2},
$$

where $\mathbf{n}=\mathbf{n}(\mathbf{x})$ denotes the unit outward normal to $\partial \Omega$ at $\mathbf{x}$. (More generally, we could have prescribed nonzero tractions of various types on $\partial \Omega_{2}$, as well as including the potential energy of body forces such as gravity in the expression for the energy (2.2), but for simplicity we have not done this, since the main difficulties we address are already present without these additions.)

To avoid interpenetration of matter, it is natural to require that $\mathbf{y}: \Omega \rightarrow$ $\mathbf{R}^{3}$ be invertible. To try to ensure that deformations have this property, we suppose that

$$
W(\mathbf{A}) \rightarrow \infty \text { as } \operatorname{det} \mathbf{A} \rightarrow 0+.
$$

So as to also prevent orientation reversal we define $W(\mathbf{A})=\infty$ if $\operatorname{det} \mathbf{A} \leq 0$. Then $W: M^{3 \times 3} \rightarrow[0, \infty]$ is continuous. Clearly if $I(\mathbf{y})<\infty$ then

$$
\operatorname{det} D \mathbf{y}(\mathbf{x})>0 \text { for a.e. } \mathbf{x} \in \Omega \text {. }
$$

Since $\mathbf{y}$ is not assumed to be $C^{1},(2.8)$ does not imply even local invertibility. For studies of local and global invertibility in the context of elasticity, or relevant to it, see Ball [1981], Bauman and Phillips [1994], Ciarlet and Nečas [1985], Fonseca and Gangbo [1995], Giaquinta, Modica and Souček [1994], Meisters and Olech [1963], Šverák [1988] and Weinstein [1985].

We assume that for any elastic material the stored-energy function $W$ is frame-indifferent, i.e.,

$$
W(\mathbf{R A})=W(\mathbf{A}) \text { for all } \mathbf{R} \in \mathrm{SO}(3), \mathbf{A} \in M^{3 \times 3} .
$$

In addition, if the material has a nontrivial isotropy group $\mathcal{S}, W$ satisfies the material symmetry condition

$$
W(\mathbf{A Q})=W(\mathbf{A}) \text { for all } \mathbf{Q} \in \mathcal{S}, \mathbf{A} \in M^{3 \times 3} .
$$

The case $\mathcal{S}=\mathrm{SO}(3)$ corresponds to an isotropic material.

For incompressible materials the deformation $\mathbf{y}$ is required to satisfy the constraint

$$
\operatorname{det} D \mathbf{y}(\mathbf{x})=1 \text { a.e. } \mathbf{x} \in \Omega \text {. }
$$

All of the problems and results contained in this article have corresponding incompressible versions, some of which we cite in the references. However, in general we do not state these explicitly. 


\subsection{Existence of equilibrium solutions}

There are two traditional routes to proving the existence of equilibrium solutions. The first, pioneered by Stoppelli [1954, 1955] and described in the book of Valent [1988], is to use the implicit function theorem in a suitable Banach space $X$ to prove the existence of an equilibrium solution close to a given one, when the data of the problem are slightly perturbed. In order to make this work, it is necessary to use spaces $X$ of sufficiently smooth mappings, for example subspaces of $W^{2, p}\left(\Omega ; \mathbf{R}^{3}\right)$ for $p>3$ or $C^{2+\alpha}\left(\Omega ; \mathbf{R}^{3}\right)$, so as to control the nonlinear dependence on $D \mathbf{y}$. In addition, the linearized elasticity operator at the given solution should be invertible as a map from $X$ to a suitable target space $Y$. While this method automatically delivers smooth solutions, it is by its nature restricted to small perturbations (for example, small boundary displacements from a stress-free state), and because of the regularity properties required for the linearized operator it in general only applies to situations when $\overline{\partial \Omega_{1}}$ and $\overline{\partial \Omega_{2}}$ do not meet, for example when one of them is empty. In particular, mixed boundary conditions typically encountered in applications, for example (2.1) with $\partial \Omega_{1}$ comprising the two end-faces of a cylindrical rod, are in general not allowed, at least with the techniques as currently developed (see Section 2.7).

The second route is to prove the existence of a global minimizer of $I$ via the direct method of the calculus of variations. In principle such a minimizer should satisfy the equilibrium equations, at least in weak form, but this turns out to be a subtle matter (see Sections 2.3, 2.4). More generally we could ask for conditions ensuring that there exist some kind of local minimizer.

Let

$$
\mathcal{A}=\left\{\mathbf{y} \in W^{1,1}\left(\Omega ; \mathbf{R}^{3}\right): I(\mathbf{y})<\infty,\left.\mathbf{y}\right|_{\partial \Omega_{1}}=\overline{\mathbf{y}}\right\},
$$

where the boundary condition is understood in the sense of trace. In the definition of $\mathcal{A}$ we could include the requirement that $y$ be invertible; this can be done in various ways, one of which is discussed in Section 2.5.

2.1 Definition. Let $1 \leq p \leq \infty$. The deformation $\mathbf{y} \in \mathcal{A}$ is a $W^{1, p}$ local minimizer of $I$ if there exists $\varepsilon>0$ such that $I(\mathbf{z}) \geq I(\mathbf{y})$ for any $\mathbf{z} \in \mathcal{A}$ with $\|\mathbf{z}-\mathbf{y}\|_{W^{1, p}} \leq \varepsilon$.

The problem of proving the existence of local, but not global, minimizers is discussed later (see Problem 9). A typical result on global minimization is the following.

2.2 Theorem. Suppose that $W$ satisfies the hypotheses

(H1) $W$ is polyconvex, i.e., $W(\mathbf{A})=g(\mathbf{A}, \operatorname{cof} \mathbf{A}, \operatorname{det} \mathbf{A})$ for all $\mathbf{A} \in M^{3 \times 3}$ for some convex $g$,

(H2) $W(\mathbf{A}) \geq c_{0}\left(|\mathbf{A}|^{2}+|\operatorname{cof} \mathbf{A}|^{3 / 2}\right)-c_{1}$ for all $\mathbf{A} \in M^{3 \times 3}$, where $c_{0}>0$.

Then, if $\mathcal{A}$ is nonempty, there exists a global minimizer $\mathbf{y}^{*}$ of $I$ in $\mathcal{A}$.

Version Dec 16, 2001 ....... Edited by wgm : Typeset on 26 January $2002-9 \mathrm{~h} 38$ 
Here and below we take $|\cdot|$ to be the Euclidean norm on $M^{3 \times 3}$ with corresponding inner product $\mathbf{A} \cdot \mathbf{B}=\operatorname{tr}\left(\mathbf{A}^{T} \mathbf{B}\right)$. Theorem 2.2 is a refinement by Müller, Qi and Yan [1994] of the result in Ball [1977a]. For the problem to be nontrivial we need that $\mathcal{H}^{2}\left(\partial \Omega_{1}\right)>0$.

The hypothesis (H1) is known to be too strong for the following reason. Let $f: M^{m \times n} \rightarrow \mathbf{R} \cup\{+\infty\}$ be Borel measurable and bounded below. We recall $f$ is said to be quasiconvex at $\mathbf{A} \in M^{m \times n}$ if the inequality

$$
\int_{\Omega} f(\mathbf{A}+D \varphi(\mathbf{x})) d \mathbf{x} \geq \int_{\Omega} f(\mathbf{A}) d \mathbf{x}
$$

holds for any $\varphi \in C_{0}^{\infty}\left(\Omega ; \mathbf{R}^{m}\right)$, and is quasiconvex if it is quasiconvex at every $\mathbf{A} \in M^{m \times n}$. Here $\Omega \subset \mathbf{R}^{n}$ is any bounded open set whose boundary $\partial \Omega$ has zero $n$-dimensional Lebesgue measure. A standard scaling argument (see, for example, Ball and Murat [1984]) shows that contrary to appearances these definitions do not depend on $\Omega$. Results of Morrey [1952] and Acerbi and Fusco [1984] imply that if $f: M^{m \times n} \rightarrow \mathbf{R}$ is quasiconvex and satisfies the growth condition

$$
C_{1}|\mathbf{A}|^{p}-C_{0} \leq f(\mathbf{A}) \leq C_{2}\left(|\mathbf{A}|^{p}+1\right) \quad \text { for all } \mathbf{A} \in M^{m \times n},
$$

where $p>1$ and where $C_{0}$ and $C_{1}>0, C_{2}>0$ are constants, then

$$
\mathcal{F}(\mathbf{y})=\int_{\Omega} f(D \mathbf{y}) d \mathbf{x}
$$

attains a global minimum on

$$
\mathcal{A}=\left\{\mathbf{y} \in W^{1,1}\left(\Omega ; \mathbf{R}^{m}\right):\left.\mathbf{y}\right|_{\partial \Omega_{1}}=\overline{\mathbf{y}}\right\} .
$$

Here we assume that $\Omega$ has Lipschitz boundary $\partial \Omega$, that $\partial \Omega_{1} \subset \partial \Omega$ is $\mathcal{H}^{n-1}$ measurable and that $\overline{\mathbf{y}}: \partial \Omega_{1} \rightarrow \mathbf{R}^{m}$ is given such that $\mathcal{A}$ is nonempty. As shown by Ball and Murat [1984], quasiconvexity of $f$ is necessary for the existence of a global minimizer for all perturbed functionals of the form

$$
\mathcal{F}_{1}(\mathbf{y})=\int_{\Omega}[f(D \mathbf{y}(\mathbf{x}))+h(\mathbf{x}, \mathbf{y}(\mathbf{x})] d \mathbf{x}
$$

with $h(\cdot, \cdot) \geq 0$ continuous. These results strongly suggest that (H1) should be replaced by the requirement that $W$ be quasiconvex, a weaker condition than polyconvexity. For example, it is easily seen that a larger class of $W$ for which Theorem 2.2 holds consists of those of the form $W=W_{1}+f$, where $W_{1}$ satisfies (H1) and (H2), and where $f: M^{3 \times 3} \rightarrow \mathbf{R}$ is quasiconvex and satisfies (2.11). That this really is a larger class can be seen by taking $f=K F$ for a large $K>0$, where $F$ is quasiconvex but not polyconvex. Such $F$ exist satisfying $F(\mathbf{R A Q})=F(\mathbf{A})$ for all $\mathbf{R}, \mathbf{Q} \in \operatorname{SO}(3), \mathbf{A} \in M^{3 \times 3}$, 
and can be constructed by the method of Šverák [1991] $]^{1}$. More generally we could take $f$ to satisfy

$$
f(\mathbf{A}) \geq C_{1}|\mathbf{A}|^{p}-C_{0}
$$

for some $p>1, C_{1}>0, C_{0}$ and to be the supremum of a nondecreasing sequence of continuous quasiconvex functions $f^{k}: M^{3 \times 3} \rightarrow[0, \infty)$, each satisfying a growth condition

$$
0 \leq \mathbf{f}^{k}(\mathbf{A}) \leq \alpha_{k}|\mathbf{A}|^{p}-\beta_{k}
$$

for constants $\alpha_{k}>0, \beta_{k}$. (Kristensen [1994] has shown that a lower semicontinuous function $f: M^{3 \times 3} \rightarrow[0, \infty]$ satisfying (2.13) is the supremum of such a sequence if and only if $f$ is closed $W^{1, p}$ quasiconvex in the sense of Pedregal [1994], namely that Jensen's inequality $\langle\nu, f\rangle \geq f(\bar{\nu})$ holds for all homogeneous $W^{1, p}$ gradient Young measures ${ }^{2} \nu$.)

However, as they stand none of the existence theorems for minimizers of integrals of general quasiconvex functions apply to elasticity, since they all assume growth conditions such as (2.11) which are not consistent with the condition (2.7). (The same applies to other results, such as the relaxation theorem of Dacorogna [1982].) In particular, it is not clear whether or not a quasiconvex $W$ satisfying our hypotheses can be written as the supremum of everywhere finite continuous quasiconvex functions. This is not true in general for quasiconvex functions $f: M^{m \times n} \rightarrow[0, \infty]$; for example we can take $m=n=2, f(\mathbf{A})=0$ if $\mathbf{A} \in\left\{\mathbf{A}_{1}, \mathbf{A}_{2}, \mathbf{A}_{3}, \mathbf{A}_{4}\right\}$ and $f(\mathbf{A})=\infty$ otherwise, where the $\mathbf{A}_{i}$ are diagonal matrices in a Tartar configuration (see Tartar [1993]), for example

$$
\begin{aligned}
& \mathbf{A}_{1}=\operatorname{diag}(-2,1), \quad \mathbf{A}_{2}=\operatorname{diag}(1,2), \\
& \mathbf{A}_{3}=\operatorname{diag}(2,-1), \quad \mathbf{A}_{4}=\operatorname{diag}(-1,-2) .
\end{aligned}
$$

${ }^{1}$ For example we can take $F=H^{\mathrm{qc}}$ to be the quasiconvexification of

$$
H(\mathbf{A})=\min \left(|\mathbf{U}(\mathbf{A})-\mathbf{1}|^{p},|\mathbf{U}(\mathbf{A})-\lambda \mathbf{1}|^{p}\right),
$$

where $\lambda>1$ and $\mathbf{U}(\mathbf{A})=\left(\mathbf{A}^{T} \mathbf{A}\right)^{1 / 2}$. The quasiconvexification $H^{\mathrm{qc}}$ of $H$ is defined to be the supremum of all quasiconvex functions $\psi \leq H$.

${ }^{2}$ See Young [1969], Tartar [1979], Ball [1989] for the definition and properties of the Young measure $\left(\nu_{\mathbf{x}}\right)_{\mathbf{x} \in \Omega}$ corresponding to a sequence of mappings $\mathbf{z}^{(j)}: \Omega \rightarrow \mathbf{R}^{s}$ satisfying a suitable bound, say $\left\|\mathbf{z}^{(j)}\right\|_{L^{1}} \leq M<\infty$, where $\Omega \subset \mathbf{R}^{n}$ is open (or measurable). For each $\mathbf{x} \in \Omega, \nu_{x}$ is a probability measure on $\mathbf{R}^{s}$ giving the limiting distribution of values of $\mathbf{z}^{(j)}(\mathbf{p})$ as $j \rightarrow \infty$ and $\mathbf{p} \rightarrow \mathbf{x}$. If $f: \mathbf{R}^{s} \rightarrow \mathbf{R}$ is continuous, then the weak limit of $f\left(z^{(j)}\right)$ in $L^{1}(E)$, where $E \subset \Omega$ is measurable, is given by the function $x \mapsto\left\langle\nu_{\mathbf{x}}, f\right\rangle$, whenever the weak limit exists. In particular, if $\mathbf{z}^{(j)} \rightarrow \mathbf{z}$ in $L^{1}(E)$, then $\mathbf{z}(\mathbf{x})=\bar{\nu}_{\mathbf{x}}$ for $\mathbf{x} \in E$, where $\bar{\mu}$ denotes the centre of mass of a measure $\mu$. Such a Young measure is homogeneous if $\nu=\nu_{\mathbf{x}}$ is independent of $\mathbf{x}$. If $1<p \leq \infty$, a $W^{1, p}$ gradient Young measure is a Young measure $\left(\nu_{\mathbf{x}}\right)_{\mathbf{x} \in \Omega}$ corresponding to a sequence $z^{(j)}=D \mathbf{y}^{(j)}$ of gradients bounded in $L^{p}\left(\Omega ; M^{m \times n}\right)$, where we identify $M^{m \times n}$ with $\mathbf{R}^{m n}$.

Version Dec 16, 2001 Edited by wgm : Typeset on 26 January $2002-9 \mathrm{~h} 38$ 
Then $f$ is quasiconvex, since any $\mathbf{y}$ with $D \mathbf{y} \in\left\{\mathbf{A}_{1}, \mathbf{A}_{2}, \mathbf{A}_{3}, \mathbf{A}_{4}\right\}$ a.e. has constant gradient (this following, for example, from the general result of Chlebík and Kirchheim [2001]). But the argument of Tartar shows that if $f$ were the supremum of continuous quasiconvex functions $f^{k}: M^{2 \times 2} \rightarrow$ $[0, \infty)$ we would have $f(0)=0$, a contradiction.

A further reason for preferring quasiconvexity to polyconvexity is that, unlike quasiconvexity, polyconvexity is not closed with respect to periodic homogenization (Braides [1994]).

Problem 1. Prove the existence of energy minimizers for elastostatics for quasiconvex stored-energy functions satisfying (2.7).

A principal difficulty here is that there is no known useful characterization of quasiconvexity. If $W$ is quasiconvex then $W$ is rank-one convex, that is the map $t \mapsto W(\mathbf{A}+t \mathbf{a} \otimes \mathbf{n})$ is convex for each $\mathbf{A} \in M^{m \times n}$ and $\mathbf{a} \in \mathbf{R}^{m}, \mathbf{n} \in \mathbf{R}^{n}$. For 40 years it seemed possible that in fact rank-one convexity was equivalent to quasiconvexity, until Šverák [1992] found his well-known counterexample for the dimensions $n \geq 2, m \geq 3$. Then Kristensen [1999] used Šverák's example to show that for the same dimensions there is no local characterization of quasiconvexity. In the absence of a characterization leading to a new proof technique, one is forced to make direct use of the definition (2.10), which leads to serious problems of approximation by piecewise affine functions when (2.7) holds.

In Ball [1977a] it was shown how the hypotheses (H1), (H2) can be satisfied for a class of isotropic materials including models of natural rubbers, via theorems exploiting the representation

$$
W(\mathbf{A})=\Phi\left(v_{1}, v_{2}, v_{3}\right)
$$

of the stored-energy function $W$ of an isotropic material, where $\Phi$ is a symmetric function of the singular values $v_{i}=v_{i}(\mathbf{A})$, that is of the eigenvalues of $\left(\mathbf{A}^{T} \mathbf{A}\right)^{1 / 2}$ (for a different proof of such theorems see Le Dret [1990]). However it is not obvious how to verify (H1) when the material is not isotropic, for example when it has cubic symmetry.

Problem 2. Are there ways of verifying polyconvexity and quasiconvexity for a useful class of anisotropic stored-energy functions?

To illustrate the difficulty in verifying (H1), in the isotropic case it is much more convenient to use the representation (2.14) rather than the equivalent representation $W(\mathbf{A})=h\left(I_{1}, I_{2}, I_{3}\right)$ in terms of the principal invariants $I_{j}=I_{j}(\mathbf{A})$. Perhaps it is significant that the function $\Phi$ in (2.14) has the same regularity as $W$, while $h$ is less regular (see Ball [1984], Sylvester [1985], Šilhavý [2000]). At any rate the more symmetric form (2.14) lends itself more easily to discussing convexity properties. For nonisotropic materials suitable representations do not seem to be available; for example, in the case of cubic symmetry it does not seem to be convenient

Version Dec 16, 2001 ....... Edited by wgm : Typeset on 26 January $2002-9$ h38 
to use the usual integrity basis (given, for example, in Green and Adkins [1970]).

\subsection{Regularity and the classification of singularities}

The main open question concerning regularity is to decide when global, or local, minimizers of $I$ are smooth. A special case is

Problem 3. When is the minimizer $\mathbf{y}^{*}$ in Theorem 2.2 smooth?

Here smooth means $C^{\infty}$ in $\Omega$, and $C^{\infty}$ up to the boundary (except in the neighbourhood of points $\mathbf{x}_{0} \in \overline{\partial \Omega_{1}} \cap \overline{\partial \Omega_{2}}$ where singularities can be expected). Clearly additional hypotheses on $W$ are needed for this to be true. One might assume, for example, that $W: M_{+}^{3 \times 3} \rightarrow \mathbf{R}$ is $C^{\infty}$, and that (H1) is strengthened by assuming $W$ to be strictly polyconvex (i.e., that $g$ is strictly convex). Also for regularity up to the boundary we would need to assume both smoothness of the boundary (except perhaps at $\overline{\partial \Omega_{1}} \cap \overline{\partial \Omega_{2}}$ ) and that $\overline{\mathbf{y}}$ is smooth. The precise nature of these extra hypotheses is to be determined. Problem 3 is unsolved even in the simplest special cases. In fact the only situation in which smoothness of $\mathbf{y}^{*}$ seems to have been proved is for the pure displacement problem ( $\partial \Omega_{2}$ empty) with small boundary displacements from a stress-free state. For this case Zhang [1991], following work of Sivaloganathan [1989], gave hypotheses under which the smooth solution to the equilibrium equations delivered by the implicit function theorem was in fact the unique global minimizer $\mathbf{y}^{*}$ of $I$ given by Theorem 2.2 .

An even more ambitious target would be to somehow classify possible singularities in minimizers of $I$ given by $(2.2)$ for generic stored-energy functions $W$. If at the same time one could associate with each such singularity a condition on $W$ that prevented it, one would also, by imposing all such conditions simultaneously, possess a set of hypotheses implying regularity. In fact it is possible to go a little way down this road. Consider first the kind of singularity frequently observed at phase boundaries in elastic crystals, in which the deformation gradient $D \mathbf{y}$ is piecewise constant, with values $\mathbf{A}, \mathbf{B}$ on either side of a plane $\{\mathbf{x} \cdot \mathbf{n}=k\}$. It was shown in Ball [1980] that, under the natural assumption that there is some matrix $\mathbf{A}_{0}$ that is a local minimizer of $W(\cdot)$, every such deformation $\mathbf{y}$ that is locally a weak solution of the Euler-Lagrange equation is trivial, that is $\mathbf{A}=\mathbf{B}$, if and only if $W$ is strictly rank-one convex (i.e., the map $t \mapsto W(\mathbf{A}+t \mathbf{a} \otimes \mathbf{n})$ is strictly convex for every $\mathbf{A}$ and all nonzero $\mathbf{a}, \mathbf{n})$. Thus strict rank-one convexity is exactly what is needed to eliminate this particular kind of singularity.

Another physically occuring singularity is that of cavitation. For radial cavitation the deformation has the form $\mathbf{y}: B(0,1) \rightarrow \mathbf{R}^{3}$, where $B(0,1)$ is the unit ball in $\mathbf{R}^{3}$, and

$$
\mathbf{y}(\mathbf{x})=r(|\mathbf{x}|) \frac{\mathbf{x}}{|\mathbf{x}|} .
$$

Version Dec 16, 2001 ....... Edited by wgm : Typeset on 26 January $2002-9$ h38 
Thus if $r(0)>0, \mathbf{y}$ is discontinuous at $\mathbf{x}=0$, where a hole of radius $r(0)$ is formed. If (H1) holds, then since polyconvexity implies quasiconvexity, the minimizer of $I$ among smooth ( $W^{1,3}$ is enough, see below) y satisfying $\mathbf{y}(\mathbf{x})=\lambda \mathbf{x}$ for $|\mathbf{x}|=1$ (i.e., $r(1)=\lambda$ ) is given by the homogeneous deformation

$$
\tilde{\mathbf{y}}_{\lambda}(\mathbf{x}) \equiv \lambda \mathbf{x}
$$

However, it was shown in Ball [1982] that for a class of stored-energy functions $W$ satisfying (H1) and the growth condition in (H2) but with $2 \leq p<3, q<\frac{3}{2}, I$ attains a minimum among radial deformations satisfying the boundary condition $\mathbf{y}(\mathbf{x})=\lambda \mathbf{x}$ for $|\mathbf{x}|=1$, and that for $\lambda>0$ sufficiently large the minimizer $\overline{\mathbf{y}}$ satisfies $r(0)>0$. Furthermore $\overline{\mathbf{y}}$ satisfies the weak form of the Euler-Lagrange equation (2.4). As a specific example we can take

$$
W(\mathbf{A})=|\mathbf{A}|^{2}+h(\operatorname{det} \mathbf{A}),
$$

for $h:(0, \infty) \rightarrow \mathbf{R}$ smooth with $h^{\prime \prime}>0, \lim _{\delta \rightarrow \infty} \frac{h(\delta)}{\delta}=\lim _{\delta \rightarrow 0+} h(\delta)=$ $\infty$. Cavitation is a common failure mechanism in polymers; for interesting pictures of almost radial cavitation of roughly spherical rubber particles imbedded in a matrix of Nylon-6 see Lazzeri and Bucknall [1995]. Müller, Qi and Yan [1994] show that if (H2) holds then no deformation with finite energy can exhibit cavitation. A somewhat stronger condition, which by the Sobolev embedding theorem obviously prevents not only cavitation but any other discontinuity in $\mathbf{y}$, is that $W(\mathbf{A}) \geq c_{0}|\mathbf{A}|^{p}-c_{1}$ for all $\mathbf{A}$, where $c_{0}>0$ and $p>3$. In fact even if $p=3$ any finite-energy deformation is continuous on account of (2.8) and the result of Vodop'yanov, Gol'dshtein and Reshetnyak [1979].

There is an extensive literature on cavitation in elasticity; a sample of the more mathematical developments can be found in the papers of Antman and Negrón-Marrero [1987], James and Spector [1991], Müller and Spector [1995], Polignone and Horgan [1993a,b], Sivaloganathan [1986, 1995, 1999], Sivaloganathan and Spector [2000a,b, 2001], Pericak-Spector and Spector [1997], Stringfellow and Abeyaratne [1989] and Stuart [1985, 1993].

An interesting feature of cavitation is that it provides a realistic example of the Lavrentiev phenomenon, whereby the infimum of the energy is different in different function spaces. Here it takes the form

$$
\inf _{\mathcal{A}_{1}} I<\inf _{\mathcal{A}_{3}} I=I\left(\tilde{\mathbf{y}}_{\lambda}\right)
$$

where $\mathcal{A}_{p}=\left\{\mathbf{y} \in W^{1, p}\left(B(0,1) ; \mathbf{R}^{3}\right):\left.\mathbf{y}\right|_{\partial B(0,1)}=\lambda \mathbf{x}\right\}$. More generally, there is a theory of minimization for elasticity with $W$ polyconvex in function spaces not allowing cavitation due to Giaquinta, Modica and Souček [1989, 1994, 1998] (see also the less technically demanding approach of Müller [1988]). Thus the same $W$ can have different minimizers in different 
function spaces; if we enlarge the space to allow not only cavitation but crack formation (see Section 2.8), then we can have different minimizers in at least three different spaces.

In cavitation there is a change of topology of the deformed configuration associated with the Lavrentiev phenomenon, but one-dimensional examples in Ball and Mizel [1985] for integrals of the form

$$
I(y)=\int_{a}^{b} f\left(x, y(x), y_{x}(x)\right) d x
$$

show that the phenomenon can occur when the minimizer $y$ is continuous with unbounded gradient. This leads to the question:

Problem 4. Can the Lavrentiev phenomenon occur for elastostatics under growth conditions on the stored-energy function ensuring that all finiteenergy deformations are continuous?

Of course if $\mathbf{y}^{*}$ is smooth then the Lavrentiev phenomenon cannot hold under the hypotheses of Theorem 2.2. Some interesting recent progress on Problem 4 is due to Foss [2001], Mizel, Foss and Hrusa [2002], who provide examples of the Lavrentiev phenomenon in two dimensions for a homogeneous isotropic polyconvex stored-energy function $W$ satisfying (2.7) and the corresponding growth condition $W(\mathbf{A}) \geq c_{0}|\mathbf{A}|^{p}-c_{1}$ for all $\mathbf{A} \in M_{+}^{2 \times 2}$ and some $p>2$. In these examples the reference configuration is given by a sector of a disk described in polar coordinates by $\Omega_{\alpha}=\{(r, \theta): 0<r<1,0<\theta<\alpha\}$, and the boundary conditions are of the 'container' type that the origin is fixed, that $\mathbf{y}\left(\Omega_{\alpha}\right) \subset \Omega_{\beta}$, and that $\mathbf{y}(1, \theta)=\left(1, \frac{\beta}{\alpha} \theta\right)$, where $0<\beta<\frac{3}{4} \alpha$. Whether such examples can be constructed for mixed boundary conditions of the type (2.1), even in two dimensions, or be associated with singularities in the interior of $\Omega$, remains open. The Lavrentiev phenomenon has important implications for the numerical computation of minimizers (see Ball [2001] and the references therein). For a useful survey of the phenomenon in one and more dimensions see Buttazzo and Belloni [1995].

There are striking examples of variational problems of the form (2.12) for which the global minimizer is not smooth. The first such example was found by Nečas [1977], who showed that if $m=n^{2}$ with $n$ sufficiently large then there exists a strictly convex $f=f(D \mathbf{y})$ whose corresponding integral

$$
\mathcal{F}(\mathbf{y})=\int_{B(0,1)} f(D \mathbf{y}) d \mathbf{x}
$$

has as global minimizer

$$
y_{i j}^{*}(\mathbf{x})=\frac{x_{i} x_{j}}{|\mathbf{x}|}, \quad \mathbf{x} \in B(0,1)
$$

subject to its own (smooth) boundary-values on $\partial B(0,1)$. Here $\mathbf{y}^{*}$ is Lipschitz but not $C^{1}$. Then Hao, Leonardi and Nečas [1996] modified the 
example to work for $n \geq 5$ with minimizer

$$
y_{i j}^{*}=\frac{x_{i} x_{j}}{|\mathbf{x}|}-\frac{1}{n}|\mathbf{x}| \delta_{i j} .
$$

By a more sophisticated method, Šverák and Yan [2000] showed that there exists a convex $f$ such that (2.17) gives a minimizer for $n=3$. In fact, working in the five-dimensional space of $3 \times 3$ traceless symmetric matrices we thus obtain an example with $n=3, m=5$. Šverák and Yan also obtained a similar example for the case $n=4, m=3$. Note that the above maps $\mathbf{y}^{*}$ are one-homogeneous, i.e., $\mathbf{y}^{*}(s \mathbf{x})=s \mathbf{y}^{*}(\mathbf{x})$ for all $s \geq 0$. In contrast Phillips [2001] has shown that when $n=2$ any one-homogeneous weak solution $\mathbf{y}$ to a strongly elliptic system of the form $\operatorname{div} \mathbf{A}(D \mathbf{y})=0$ is linear.

Even if $\mathbf{y}^{*}$ is not smooth everywhere, we can ask for smoothness outside a closed set of Lebesgue measure zero. That such a result is true is strongly suggested by the classical partial regularity theorem of Evans [1986], which (with the incorporation of a weakening of the growth condition due to Acerbi and Fusco [1988]) states that any global minimizer has this property for a class of integrals of the form (2.16) with $f$ satisfying (2.11) and the strong quasiconvexity condition that for some $p \geq 2$

$$
\int_{\Omega}[f(\mathbf{A}+D \varphi)-f(\mathbf{A})] d \mathbf{x} \geq \gamma \int_{\Omega}\left[|D \varphi|^{2}+|D \varphi|^{p}\right] d \mathbf{x},
$$

for all $\mathbf{A} \in M^{m \times n}$, all $\varphi \in C_{0}^{\infty}\left(\Omega ; \mathbf{R}^{m}\right)$. Recently, Kristensen and Taheri [2001] have proved the same result but for $W^{1, p}$ local minimizers. However, it is not known how to extend these theorems to the case of elasticity when (2.7) holds (see Ball [1998] for a brief discussion).

In proving regularity or partial regularity, it is not sufficient to just use the fact (if it is a fact, see below) that $\mathbf{y}^{*}$ satisfies the weak form of the Euler-Lagrange equation. This follows from the example of Müller and Šverák [2001] of a Lipschitz mapping $\mathbf{y}: \Omega \rightarrow \mathbf{R}^{2}$, with $\Omega \subset \mathbf{R}^{2}$ a bounded domain, that is nowhere $C^{1}$ and solves the weak form of the Euler-Lagrange equation for an integral

$$
I(\mathbf{y})=\int_{\Omega} F(D \mathbf{y}) d \mathbf{x}
$$

with $F$ strictly quasiconvex. As shown by Kristensen and Taheri [2001] y can even be a $W^{1, \infty}$ local minimizer.

There seems to be little indication from experiment that natural rubbers have equilibrium solutions with singularities other than those involving cavitation or other forms of fracture. Thus it seems a reasonable conjecture that minimizers are smooth for models of natural rubber for which the stored-energy function satisfies growth conditions prohibiting discontinuities for deformations of finite energy. In view of the above and other 
counterexamples for elliptic systems, if minimizers are smooth it must be for special reasons applying to elasticity. Three plausible such reasons are: (a) the fact that the integrand $W(D \mathbf{y})$ does not depend explicitly on $\mathbf{y}$ (though dependence on $\mathbf{x}$ is allowed), (b) the fact that the dimensions $m=n=3$ are low, (c) the frame-indifference of $W$ (see (2.9)). A fourth possible reason is (d) invertibility of $\mathbf{y}$, which we discuss now.

That invertibility could have an effect on regularity was first shown by Bauman, Owen and Phillips [1991], who gave an example of an essentially two-to-one equilibrium solution in a ball for two-dimensional elasticity, with stored-energy function of the form (2.15), that is $C^{1}$ but not smooth, and is such that det $D \mathbf{y}$ vanishes at the centre of the ball. An instructive example (resulting from discussions with X. Yan and J. Bevan) is that of minimizing the two-dimensional energy for an incompressible material

$$
I(\mathbf{y})=\int_{B}|D \mathbf{y}|^{2} d \mathbf{x}
$$

where $B=B(0,1)$ is the unit disc in $\mathbf{R}^{2}$, and $\mathbf{y}: B \rightarrow \mathbf{R}^{2}$, in the set of admissible mappings

$$
\mathcal{A}=\left\{\mathbf{y} \in W^{1,2}\left(B ; \mathbf{R}^{2}\right): \operatorname{det} D \mathbf{y}=1 \text { a.e. },\left.\mathbf{y}\right|_{\partial B}=\overline{\mathbf{y}}\right\},
$$

where in polar coordinates $\overline{\mathbf{y}}:(r, \theta) \mapsto\left(\frac{1}{\sqrt{2}} r, 2 \theta\right)$. Then there exists a global minimizer $y^{*}$ of $I$ in $\mathcal{A}$. (Note that $\mathcal{A}$ is nonempty since $\overline{\mathbf{y}} \in \mathcal{A}$.) But since by degree theory there are no $C^{1}$ maps y satisfying the boundary condition (2.18), it is immediate that $y^{*}$ is not $C^{1}$.

For interesting maximum principles satisfied by smooth equilibrium solutions in two-dimensional elasticity, with stored-energy function of the form (2.15), see Bauman, Owen and Phillips [1991a, 1992].

\subsection{Satisfaction of the Euler-Lagrange equation and uniform positivity of the Jacobian.}

Here we return to the computation formally leading to the weak form (2.4) of the Euler-Lagrange equation, under the assumption (2.7). If $\mathbf{y}^{*} \in$ $W^{1, \infty}\left(\Omega ; \mathbf{R}^{3}\right)$ is a $W^{1, \infty}$ local minimizer of $I$ in

$$
\mathcal{A}=\left\{\mathbf{y} \in W^{1,1}\left(\Omega ; \mathbf{R}^{3}\right):\left.\mathbf{y}\right|_{\partial \Omega_{1}}=\overline{\mathbf{y}}\right\}
$$

and if (2.8) holds in the stronger form that for some $\varepsilon>0$

$$
\operatorname{det} D \mathbf{y}^{*}(\mathbf{x}) \geq \varepsilon \text { for a.e. } \mathbf{x} \in \Omega,
$$

then it is easily seen that $\mathbf{y}^{*}$ satisfies (2.4). In fact we can then pass to the limit $\tau \rightarrow 0$ using bounded convergence in the difference quotient

$$
\int_{\Omega} \frac{1}{\tau}\left[W\left(D \mathbf{y}^{*}+\tau D \varphi\right)-W\left(D \mathbf{y}^{*}\right)\right] d x
$$

Version Dec 16, 2001

Edited by wgm : Typeset on 26 January $2002-9$ h 38 
since by (2.19) we have $\operatorname{det}\left(D \mathbf{y}^{*}(\mathbf{x})+\tau D \varphi(\mathbf{x})\right) \geq \varepsilon / 2$ for a.e. $\mathbf{x} \in \Omega$. However, if only (2.7) is assumed, or if $\mathbf{y}^{*}$ is not assumed in advance to be in $W^{1, \infty}\left(\Omega ; \mathbf{R}^{3}\right)$, then it is not obvious how to pass to the limit.

Problem 5. Prove or disprove that, under reasonable growth conditions on $W$, global or suitably defined local minimizers of I satisfy the weak form (2.4) of the Euler-Lagrange equation.

Problem 6. Prove or disprove that, under reasonable growth conditions on $W$, global or suitably defined local minimizers of I satisfy (2.19).

If $W(\mathbf{A}) \rightarrow \infty$ as $|\mathbf{A}| \rightarrow \infty$ and if $\mathbf{y}^{*} \notin W^{1, \infty}$, or if (2.19) does not hold, then $W\left(D \mathbf{y}^{*}\right)$ is essentially unbounded. This is at first sight inconsistent with $\mathbf{y}^{*}$ being a minimizer, but we know from the one-dimensional examples in Ball and Mizel [1985] and from the example of cavitation that it can pay to have the integrand infinite somewhere so that it is smaller somewhere else. In general, it is not possible to estimate the integrand in the difference quotient (2.20) in terms of $W\left(D \mathbf{y}^{*}\right)$, the only relevant quantity that is obviously integrable. This difficulty was pointed out by Antman [1976], who was the first to address the issue of satisfaction of the Euler-Lagrange equation for one-dimensional problems of elasticity when (2.7) holds; in this context a device essentially due to Tonelli [1921] can be used to prove that the Euler-Lagrange equation holds (see also Ball [1981a]) without any supplementary growth conditions on $W$.

It is perhaps worth making the simple observation that a smooth deformation y may satisfy $I(\mathbf{y})<\infty$ and $\operatorname{det} D \mathbf{y}(\mathbf{x})>0$ a.e. without (2.19) holding. As an example we may take $\Omega=B(0,1)$ and

$$
\mathbf{y}(\mathbf{x})=|\mathbf{x}|^{2} \mathbf{x}
$$

with $W(\mathbf{A})=-\log \operatorname{det} \mathbf{A}+g(\mathbf{A})$, where $g: M^{3 \times 3} \rightarrow \mathbf{R}$ is smooth.

For a class of strongly elliptic stored-energy functions having the form $W(\mathbf{A})=g(\mathbf{A})+h(\operatorname{det} \mathbf{A})$, where $g: M^{3 \times 3} \rightarrow \mathbf{R}$ and $h:(0, \infty) \rightarrow[0, \infty)$ are smooth with $h(\delta) \rightarrow \infty$ as $\delta \rightarrow 0+$ at a polynomial rate, Bauman, Owen and Phillips [1991] show that if $\mathbf{y} \in C^{1, \beta}$ satisfies the energy-momentum weak form of the Euler-Lagrange equation in (2.22) below, then in fact $\mathbf{y}$ is a smooth solution of the Euler-Lagrange equation (2.5) and the strict positivity condition (2.19) holds.

As was pointed out in Ball [1984a], it is possible to derive different first-order necessary conditions for a minimizer when (2.7) holds. (Later Giaquinta, Modica and Souček [1989] derived the same first-order conditions in their framework of Cartesian currents, under somewhat stronger hypotheses.) We give here improved versions of these results.

We consider the following conditions that may be satisfied by $W$ :

(C1) $\left|D_{\mathbf{A}} W(\mathbf{A}) \mathbf{A}^{T}\right| \leq K(W(\mathbf{A})+1)$ for all $\mathbf{A} \in M_{+}^{3 \times 3}$, where $K>0$ is a constant, and

Version Dec 16, 2001 ....... Edited by wgm : Typeset on 26 January $2002-9$ h38 
(C2) $\left|\mathbf{A}^{T} D_{\mathbf{A}} W(\mathbf{A})\right| \leq K(W(\mathbf{A})+1)$ for all $\mathbf{A} \in M_{+}^{3 \times 3}$, where $K>0$ is a constant.

As usual, | • | denotes the Euclidean norm on $M^{3 \times 3}$, for which the inequalities $|\mathbf{A} \cdot \mathbf{B}| \leq|\mathbf{A}| \cdot|\mathbf{B}|$ and $|\mathbf{A B}| \leq|\mathbf{A}| \cdot|\mathbf{B}|$ hold. But of course the conditions are independent of the norm used up to a possible change in the constant $K$.

2.3 Proposition. Let $W$ satisfy (C2). Then $W$ satisfies (C1).

Proof. Since $W$ is frame-indifferent the matrix $D_{\mathbf{A}} W(\mathbf{A}) \mathbf{A}^{T}$ is symmetric (this is equivalent to the symmetry of the Cauchy stress tensor-see (2.27) below). Hence

$$
\begin{aligned}
\left|D_{\mathbf{A}} W(\mathbf{A}) \mathbf{A}^{T}\right|^{2} & =\left[D_{\mathbf{A}} W(\mathbf{A}) \mathbf{A}^{T}\right] \cdot\left[\mathbf{A}\left(D_{\mathbf{A}} W(\mathbf{A})\right)^{T}\right] \\
& =\left[\mathbf{A}^{T} D_{\mathbf{A}} W(\mathbf{A})\right] \cdot\left[\mathbf{A}^{T} D_{\mathbf{A}} W(\mathbf{A})\right]^{T} \\
& \leq\left|\mathbf{A}^{T} D_{\mathbf{A}} W(\mathbf{A})\right|^{2}
\end{aligned}
$$

from which the result follows.

Example. Let

$$
W(\mathbf{A})=\left(\mathbf{A}^{T} \mathbf{A}\right)_{11}+\frac{1}{\operatorname{det} \mathbf{A}} .
$$

Then $W$ satisfies (C1) and (2.9), but not (C2).

2.4 Theorem. For some $1 \leq p<\infty$ let $\mathbf{y} \in \mathcal{A} \cap W^{1, p}\left(\Omega ; \mathbf{R}^{3}\right)$ be a $W^{1, p}$ local minimizer of $I$ in $\mathcal{A}$.

(i) Let $W$ satisfy (C1). Then

$$
\int_{\Omega}\left[D_{\mathbf{A}} W(D \mathbf{y}) D \mathbf{y}^{T}\right] \cdot D \varphi(\mathbf{y}) d \mathbf{x}=0
$$

for all $\varphi \in C^{1}\left(\mathbf{R}^{3} ; \mathbf{R}^{3}\right)$ such that $\varphi$ and $D \varphi$ are uniformly bounded and satisfy $\left.\varphi(\mathbf{y})\right|_{\partial \Omega_{1}}=0$ in the sense of trace.

(ii) Let $W$ satisfy $(\mathrm{C} 2)$. Then

$$
\int_{\Omega}\left[W(D \mathbf{y}) \mathbf{1}-D \mathbf{y}^{T} D_{\mathbf{A}} W(D \mathbf{y})\right] \cdot D \varphi d \mathbf{x}=0
$$

for all $\varphi \in C_{0}^{1}\left(\Omega ; \mathbf{R}^{3}\right)$.

We use the following simple lemma.

2.5 Lemma. (a) If $W$ satisfies (C1) then there exists $\gamma>0$ such that if

$$
\mathbf{C} \in M_{+}^{3 \times 3} \quad \text { and } \quad|\mathbf{C}-\mathbf{1}|<\gamma
$$

then

$$
\left|D_{\mathbf{A}} W(\mathbf{C A}) \mathbf{A}^{T}\right| \leq 3 K(W(\mathbf{A})+1) \quad \text { for all } \mathbf{A} \in M_{+}^{3 \times 3}
$$


(b) If $W$ satisfies (C2) then there exists $\gamma>0$ such that if $\mathbf{C} \in M_{+}^{3 \times 3}$ and $|\mathbf{C}-\mathbf{1}|<\gamma$ then

$$
\left|\mathbf{A}^{T} D_{\mathbf{A}} W(\mathbf{A C})\right| \leq 3 K(W(\mathbf{A})+1) \quad \text { for all } \mathbf{A} \in M_{+}^{3 \times 3} .
$$

Proof. We prove (a); the proof of (b) is similar. We first show that there exists $\gamma>0$ such that if $|\mathbf{C}-\mathbf{1}|<\gamma$ then

$$
W(\mathbf{C A})+1 \leq \frac{3}{2}(W(\mathbf{A})+1) \quad \text { for all } \mathbf{A} \in M_{+}^{3 \times 3} .
$$

For $t \in[0,1]$ let $\mathbf{C}(t)=t \mathbf{C}+(1-t) \mathbf{1}$. Choose $\gamma \in\left(0, \frac{1}{6 K}\right)$ sufficiently small so that $|\mathbf{C}-\mathbf{1}|<\gamma$ implies that $\left|\mathbf{C}(t)^{-1}\right| \leq 2$ for all $t \in[0,1]$. This is possible since $|\mathbf{1}|=\sqrt{3}<2$. For $|\mathbf{C}-\mathbf{1}|<\gamma$ we have that

$$
\begin{aligned}
W(\mathbf{C A})-W(\mathbf{A}) & =\int_{0}^{1} \frac{d}{d t} W(\mathbf{C}(t) \mathbf{A}) d t \\
& =\int_{0}^{1} D_{\mathbf{A}} W(\mathbf{C}(t) \mathbf{A}) \cdot[(\mathbf{C}-\mathbf{1}) \mathbf{A}] d t \\
& =\int_{0}^{1} D_{\mathbf{A}} W(\mathbf{C}(t) \mathbf{A})(\mathbf{C}(t) \mathbf{A})^{T} \cdot\left((\mathbf{C}-\mathbf{1}) \mathbf{C}(t)^{-1}\right) d t \\
& \leq K \int_{0}^{1}[W(\mathbf{C}(t) \mathbf{A})+1] \cdot|\mathbf{C}-\mathbf{1}| \cdot\left|\mathbf{C}(t)^{-1}\right| d t \\
& \leq 2 K \gamma \int_{0}^{1}(W(\mathbf{C}(t) \mathbf{A})+1) d t .
\end{aligned}
$$

Let $\theta(\mathbf{A})=\sup _{|\mathbf{C}-\mathbf{1}|<\gamma} W(\mathbf{C A})$. Then

$$
W(\mathbf{C A})-W(\mathbf{A}) \leq \theta(\mathbf{A})-W(\mathbf{A}) \leq 2 K \gamma(\theta(\mathbf{A})+1),
$$

from which (2.24) follows.

Finally, if $|\mathbf{C}-\mathbf{1}|<\gamma$ we have from (C1) and (2.24) that

$$
\begin{aligned}
\left|D_{\mathbf{A}} W(\mathbf{C A}) \mathbf{A}^{T}\right| & =\left|D_{\mathbf{A}} W(\mathbf{C A})(\mathbf{C A})^{T} \mathbf{C}^{-T}\right| \\
& \leq K(W(\mathbf{C A})+\mathbf{1})\left|\mathbf{C}^{-T}\right| \\
& \leq 3 K(W(\mathbf{A})+1)
\end{aligned}
$$

as required.

Proof of Theorem 2.4. (i) Given $\varphi$ as in the theorem, define for $|\tau|$ sufficiently small

$$
\mathbf{y}_{\tau}(\mathbf{x}):=\mathbf{y}(\mathbf{x})+\tau \varphi(\mathbf{y}(\mathbf{x}))
$$

Then $\mathbf{y}_{\tau} \in \mathcal{A}$ and

$$
D \mathbf{y}_{\tau}(\mathbf{x})=(\mathbf{1}+\tau D \varphi(\mathbf{y}(\mathbf{x}))) D \mathbf{y}(\mathbf{x}) \text { a.e. } \mathbf{x} \in \Omega .
$$

Version Dec 16, 2001 Edited by wgm : Typeset on 26 January $2002-9$ h 38 
In particular $\operatorname{det} D \mathbf{y}_{\tau}(\mathbf{x})>0$ for a.e. $\mathbf{x} \in \Omega$ and $\lim _{\tau \rightarrow 0}\left\|\mathbf{y}_{\tau}-\mathbf{y}\right\|_{W^{1, p}}=0$. Hence $I\left(\mathbf{y}_{\tau}\right) \geq I(\mathbf{y})$ for $|\tau|$ sufficiently small. But

$$
\begin{aligned}
& \frac{1}{\tau}\left(I\left(\mathbf{y}_{\tau}\right)-I(\mathbf{y})\right) \\
& \quad=\frac{1}{\tau} \int_{\Omega} \int_{0}^{1} \frac{d}{d s} W((\mathbf{1}+s \tau D \varphi(\mathbf{y}(\mathbf{x}))) D \mathbf{y}(\mathbf{x})) d s d \mathbf{x} \\
& \quad=\int_{\Omega} \int_{0}^{1} D W((\mathbf{1}+s \tau D \varphi(\mathbf{y}(\mathbf{x}))) D \mathbf{y}(\mathbf{x})) \cdot[D \varphi(\mathbf{y}(\mathbf{x})) D \mathbf{y}(\mathbf{x})] d s d \mathbf{x} .
\end{aligned}
$$

Since by Lemma 2.5(a) the integrand is bounded by the integrable function

$$
3 K(W(D \mathbf{y}(\mathbf{x}))+1) \sup _{\mathbf{z} \in \mathbf{R}^{3}}|D \varphi(\mathbf{z})|,
$$

we may pass to the limit $\tau \rightarrow 0$ using dominated convergence to obtain $(2.21)$.

(ii) This follows in a similar way to (i) from Lemma 2.5 (b). Since most of the details have already been written down in Bauman, Owen and Phillips [1991a] we just sketch the idea. Let $\varphi \in C_{0}^{1}\left(\Omega ; \mathbf{R}^{3}\right)$. For sufficiently small $\tau>0$ the mapping $\theta_{\tau}$ defined by

$$
\theta_{\tau}(\mathbf{z}):=\mathbf{z}+\tau \varphi(\mathbf{z})
$$

belongs to $C^{1}\left(\Omega ; \mathbf{R}^{3}\right)$, satisfies $\operatorname{det} D \theta_{\tau}(\mathbf{z})>0$, and coincides with the identity on $\partial \Omega$. Standard arguments from degree theory imply that $\theta_{\tau}$ is a diffeomorphism of $\Omega$ to itself. Thus the 'inner variation'

$$
\mathbf{y}_{\tau}(\mathbf{x}):=\mathbf{y}\left(\mathbf{z}_{\tau}\right), \quad \mathbf{x}=\mathbf{z}_{\tau}+\tau \varphi\left(\mathbf{z}_{\tau}\right)
$$

defines a mapping $\mathbf{y}_{\tau} \in \mathcal{A}$, and

$$
D \mathbf{y}_{\tau}(x)=D \mathbf{y}\left(\mathbf{z}_{\tau}\right)\left[\mathbf{1}+\tau D \varphi\left(\mathbf{z}_{\tau}\right)\right]^{-1} \quad \text { a.e. } \mathbf{x} \in \Omega .
$$

Since $\mathbf{y} \in W^{1, p}$ it follows easily that $\left\|\mathbf{y}_{\tau}-\mathbf{y}\right\|_{W^{1, p}} \rightarrow 0$ as $\tau \rightarrow 0$. Changing variables we obtain

$$
I\left(\mathbf{y}_{\tau}\right)=\int_{\Omega} W\left(D \mathbf{y}(\mathbf{z})[\mathbf{1}+\tau D \varphi(\mathbf{z})]^{-1}\right) \operatorname{det}(\mathbf{1}+\tau D \varphi(\mathbf{z})) d \mathbf{z},
$$

from which (2.22) follows using (2.23) and dominated convergence.

In order to give an interpretation of Theorem 2.4 (i), let us make the following

Invertibility Hypothesis. $\quad \mathbf{y}$ is a homeomorphism of $\Omega$ onto $\Omega^{\prime}:=\mathbf{y}(\Omega)$, $\Omega^{\prime}$ is a bounded domain, and the change of variables formula

$$
\int_{\Omega} f(\mathbf{y}(\mathbf{x})) \operatorname{det} D \mathbf{y}(\mathbf{x}) d \mathbf{x}=\int_{\Omega^{\prime}} f(\mathbf{z}) d \mathbf{z}
$$

holds whenever $f: \mathbf{R}^{3} \rightarrow \mathbf{R}$ is measurable, provided that one of the integrals in (2.25) exists. 
Sufficient conditions for this hypothesis to hold are given in Ball [1981] and Šverák [1988].

2.6 Theorem. Assume that the hypotheses of Theorem 2.4 (i) and the Invertibility Hypothesis hold. Then

$$
\int_{\Omega^{\prime}} \sigma(\mathbf{z}) \cdot D \varphi(\mathbf{z}) d \mathbf{z}=0
$$

for all $\varphi \in C^{1}\left(\mathbf{R}^{3} ; \mathbf{R}^{3}\right)$ such that $\left.\varphi\right|_{\mathbf{y}\left(\partial \Omega_{1}\right)}=0$, where the Cauchy stress tensor $\sigma$ is defined by

$$
\sigma(\mathbf{z}):=\mathbf{T}\left(\mathbf{y}^{-1}(\mathbf{z})\right), \quad z \in \Omega^{\prime}
$$

and

$$
\mathbf{T}(\mathbf{x})=(\operatorname{det} D \mathbf{y}(\mathbf{x}))^{-1} D_{\mathbf{A}} W(D \mathbf{y}(\mathbf{x})) D \mathbf{y}(\mathbf{x})^{T} .
$$

Proof. Since by assumption $\mathbf{y}(\bar{\Omega})$ is bounded, we can assume that $\varphi$ and $D \varphi$ are uniformly bounded. Thus (2.26) follows immediately from (2.21), (2.25) and (2.27).

Thus Theorem 2.4(i) asserts that $\mathbf{y}$ satisfies the spatial (Eulerian) form of the equilibrium equations. Theorem 2.4 (ii), on the other hand, involves the so-called energy-momentum tensor $W(\mathbf{A}) \mathbf{1}-\mathbf{A}^{T} D_{\mathbf{A}} W(\mathbf{A})$, and is a multi-dimensional version of the Du Bois Reymond or Erdmann equation of the one-dimensional calculus of variations.

The hypotheses $(\mathrm{C} 1)$ and $(\mathrm{C} 2)$ imply that $W$ has polynomial growth. More precisely, we have

2.7 Proposition. Suppose $W$ satisfies $(\mathrm{C} 1)$ or $(\mathrm{C} 2)$. Then for some $s>0$.

$$
W(\mathbf{A}) \leq M\left(|\mathbf{A}|^{s}+\left|\mathbf{A}^{-1}\right|^{s}\right) \text { for all } \mathbf{A} \in M_{+}^{3 \times 3} \text {. }
$$

Proof. Let $\mathbf{V} \in M^{3 \times 3}$ be symmetric. For $t \geq 0$

$$
\begin{aligned}
\left|\frac{d}{d t} W\left(e^{t \mathbf{V}}\right)\right| & =\left|\left(D_{\mathbf{A}} W\left(e^{t \mathbf{V}}\right) e^{t \mathbf{V}}\right) \cdot \mathbf{V}\right| \\
& =\left|\left(e^{t \mathbf{V}} D_{\mathbf{A}} W\left(e^{t \mathbf{V}}\right)\right) \cdot \mathbf{V}\right| \\
& \leq K\left(W\left(e^{t \mathbf{V}}\right)+1\right)|\mathbf{V}|
\end{aligned}
$$

From this it follows that

$$
W\left(e^{\mathbf{v}}\right)+1 \leq(W(\mathbf{1})+1) e^{K|\mathbf{V}|} .
$$

Now set $\mathbf{V}=\ln \mathbf{U}$, where $\mathbf{U}=\mathbf{U}^{T}>0$, and denote by $v_{i}$ the eigenvalues of U. Since

$$
\begin{aligned}
|\ln \mathbf{U}| & =\left(\sum_{i=1}^{3}\left(\ln v_{i}\right)^{2}\right)^{1 / 2} \\
& \leq \sum_{i=1}^{3}\left|\ln v_{i}\right|,
\end{aligned}
$$

Version Dec 16, 2001 Edited by wgm : Typeset on 26 January $2002-9 \mathrm{~h} 38$ 
it follows that

$$
\begin{aligned}
e^{K|\ln \mathbf{U}|} & \leq\left(v_{1}^{K}+v_{1}^{-K}\right)\left(v_{2}^{K}+v_{2}^{-K}\right)\left(v_{3}^{K}+v_{3}^{-K}\right) \\
& \leq 3^{-3}\left(\sum_{i=1}^{3} v_{i}^{K}+\sum_{i=1}^{3} v_{i}^{-K}\right)^{1 / 3} \\
& \leq C\left(\sum_{i=1}^{3} v_{i}^{3 K}+\sum_{i=1}^{3} v_{i}^{-3 K}\right)^{3} \\
& \leq C_{1}\left[|\mathbf{U}|^{3 K}+\left|\mathbf{U}^{-1}\right|^{3 K}\right]
\end{aligned}
$$

where $C>0, C_{1}>0$ are constants. From (2.29) we thus obtain

$$
W(\mathbf{U}) \leq M\left(|\mathbf{U}|^{3 K}+\left|\mathbf{U}^{-1}\right|^{3 K}\right),
$$

where $M=C_{1}(W(\mathbf{1})+1)$. The result now follows from the polar decomposition $\mathbf{A}=\mathbf{R U}$ of an arbitrary $\mathbf{A} \in M_{+}^{3 \times 3}$, where $\mathbf{R} \in \mathrm{SO}(3)$, $\mathbf{U}=\mathbf{U}^{T}>0$.

It is easily seen that if $W$ is isotropic then both (C1) and (C2) are equivalent to the condition that

$$
\left|\left(v_{1} \Phi_{, 1}, v_{2} \Phi, 2, v_{3} \Phi_{, 3}\right)\right| \leq K\left(\Phi\left(v_{1}, v_{2}, v_{3}\right)+1\right)
$$

for all $v_{i}>0$ and some $K>0$, where $\Phi$ is given by (2.14) and $\Phi_{, i}=\partial \Phi / \partial v_{i}$. In particular, both $(\mathrm{C} 1)$ and $(\mathrm{C} 2)$ hold for the class of Ogden materials (Ogden $[1972 \mathrm{a}, \mathrm{b}]$ ), for which $\Phi$ has the form

$$
\Phi\left(v_{1}, v_{2}, v_{3}\right)=\sum_{i=1}^{M} a_{i} \varphi\left(\alpha_{i}\right)+\sum_{i=1}^{N} b_{i} \psi\left(\beta_{i}\right)+h\left(v_{1} v_{2} v_{3}\right)
$$

where

$$
\varphi(\alpha)=v_{1}^{\alpha}+v_{2}^{\alpha}+v_{3}^{\alpha}, \quad \psi(\beta)=\left(v_{2} v_{3}\right)^{\beta}+\left(v_{3} v_{1}\right)^{\beta}+\left(v_{1} v_{2}\right)^{\beta}
$$

$a_{i}>0, b_{i}>0, \alpha_{i} \neq 0, \beta_{i} \neq 0$, and where $h:(0, \infty) \rightarrow[0, \infty)$ is convex, with $h(\delta) \rightarrow \infty$ as $\delta \rightarrow 0$, provided that $\left|\delta h^{\prime}(\delta)\right| \leq K_{1}(h(\delta)+1)$ for all $\delta>0$.

\subsection{Regularity and self-contact}

An interesting approach to the problem of invertibility in mixed boundaryvalue problems (i.e., to the non-interpenetration of matter) is due to Ciarlet and Nečas [1985]. They proposed minimizing

$$
I(\mathbf{y})=\int_{\Omega} W(D \mathbf{y}) d \mathbf{x}
$$

Version Dec 16, 2001

Edited by wgm : Typeset on 26 January $2002-9$ h 38 
subject to the boundary condition (2.1) and the global constraint

$$
\int_{\Omega} \operatorname{det} D \mathbf{y}(\mathbf{x}) d \mathbf{x} \leq \text { volume }(\mathbf{y}(\Omega)),
$$

and they gave hypotheses under which the minimum was attained, these hypotheses being weakened by Qi [1988]. They further showed that any minimizer is one-to-one almost everywhere, and that assuming sufficient regularity of the free boundary $\mathbf{y}\left(\partial \Omega_{2}\right)$ the tangential components of the normal stress vector vanish there. Consequently they identified the above constrained boundary-value problem as corresponding to the case of smooth (i.e., frictionless) self-contact. A related but somwhat different formulation has recently been proposed by Pantz [2001a]; see also Giaquinta, Modica and Souček [1994].

Problem 7. Justify the Ciarlet-Nečas minimization problem, or an appropriate modification of it, as a model of smooth self-contact.

The problem here is to construct suitable variations in the neighbourhood of a region of self-contact of a minimizer to establish that in some sense the tangential stress components vanish there. This is non-trivial because in principle the two parts of the boundary in contact with one another could be wildly deformed and interlocked in a very complex configuration. If such a result could be obtained, a more ambitious target would be to establish the regularity properties of the free boundary in both the self-contacting and non self-contacting regions.

\subsection{Uniqueness of solutions}

For mixed boundary-value problems of elasticity nonuniqueness of equilibrium solutions is common-place, the most familiar examples being those associated with buckling of rods, plates and shells. Buckling can occur even for pure zero-traction boundary conditions, such as in the eversion of part of a spherical shell. For the pure zero-traction problem one can even have nonuniqueness among homogeneous dilatations (see Ball [1982]). Nonuniqueness of these types is expected to hold, and to some extent can be proved rigorously, when the stored-energy function satisfies favourable hypotheses such as strict polyconvexity (though see Section 2.7). For storedenergy functions corresponding to elastic crystals, for which there are many minimum energy configurations with a continuum of different sets of phase boundaries, the extent of non-uniqueness is of course much greater.

For pure displacement boundary conditions, with a strictly polyconvex (or strictly quasiconvex) stored-energy function satisfying favourable growth conditions, the situation as regards uniqueness is less clear. John [1972b] proved uniqueness for smooth deformations with uniformly small strains (but possibly large rotations). In the same paper he gave a heuristic

Version Dec 16, 2001 ....... Edited by wgm : Typeset on 26 January $2002-9$ h38 
counter-example to uniqueness for the case of an annular two-dimensional body, and this has been made rigorous by Post and Sivaloganathan [1997] (see Section 2.7), who also proved nonuniquenesss for an analogous threedimensional problem with $\Omega$ a torus. But what if $\Omega$ is homeomorphic to a ball? In this case we have already seen that cavitation provides one counterexample to uniqueness, though the cavitating solution is discontinuous.

Problem 8. Prove or disprove the uniqueness of sufficiently smooth equilibrium solutions to pure displacement boundary-value problems for homogeneous bodies when the stored-energy function $W$ is strictly polyconvex and $\Omega$ is homeomorphic to a ball.

The answer to this problem probably depends on both the geometry of $\Omega$ and the boundary conditions. For example, suppose that $\Omega$ is a ball, and that the boundary conditions correspond to severely squeezing the ball until it has a dumb-bell shape consisting of two roughly ball-shaped regions connected by a narrow passage. In this case one might expect, though it is not obvious how to prove it, that there might be equilibrium solutions in which material from one half of $\Omega$ is pulled through into the other half, but prevented from returning by the constriction. On the other hand, an elegant result of Knops and Stuart [1984] implies uniqueness for the case when the boundary displacements are linear and $\Omega$ is star-shaped (see also Taheri [2001b]).

\subsection{Structure of the solution set}

Problem 9. Devise general methods for proving the existence of local minimizers of I that are not global minimizers, and of other weak solutions of the equilibrium equations.

For the existence of local minimizers there are two natural approaches. First we could try to use the direct method of the calculus of variations in a suitable subset of $\mathcal{A}$. For example, under the hypotheses of Theorem 2.2 suppose that we want to prove the existence of a local minimizer with respect to some metric $d$ on $\mathcal{A}$. Assume that $d$ is such that if $z^{(j)} \in \mathcal{A}$ with $z^{(j)} \rightarrow z$ in $W^{1,1}\left(\Omega ; \mathbf{R}^{3}\right)$ and $\sup I\left(z^{(j)}\right)<\infty$ then $d\left(z^{(j)}, z\right) \rightarrow 0$.

Let $U \subset \mathcal{A}$ be open with respect to $d$, with closure $\bar{U}$ and boundary $\partial U$. By the direct method, $I$ attains a minimum $\hat{y}$ on $\bar{U}$. Suppose now that we can prove that

$$
\inf _{\partial U} I>\inf _{U} I>\inf _{\mathcal{A}} I
$$

Then $\hat{y} \in U$ and is a local, but not global, minimizer with respect to $d$. I believe that it should be possible to implement this method in some realistic examples, but have not seen it done. The only results on local minimizers in nonlinear elasticity using the direct method that I am aware of are due to

Version Dec 16, 2001 ....... Edited by wgm : Typeset on 26 January $2002-9$ h38 
Post and Sivaloganathan [1997], who prove the existence of local but not global minimizers for certain two-dimensional problems (see Section 2.6) for which the domain $\Omega$ has nontrivial topology by global minimization in a weakly closed homotopy class, and to Taheri [2001a], who generalizes the results in Post and Sivaloganathan [1997] to a wider class of domains.

The second approach is to find by some method a sufficiently smooth solution $\hat{y}$ to the equilibrium equations and attempt to show directly that it is a local minimizer. For local minimizers in $W^{1, \infty}\left(\Omega ; \mathbf{R}^{3}\right)$ (weak local minimizers) this can be done in principle by checking positivity of the second variation. However for local minimizers in $W^{1, p}\left(\Omega ; \mathbf{R}^{3}\right)$ with $1 \leq p<\infty$, or in $L^{q}\left(\Omega ; \mathbf{R}^{3}\right), 1 \leq q \leq \infty$, the task is made much more difficult by the absence of a known generalization to higher dimensions of the Weierstrass fundamental sufficiency theorem of the one-dimensional calculus of variations (for a discussion see Ball [1998]). Sometimes it is possible to circumvent the lack of such a theory. For example, in a dead-load traction problem arising from the bi-axial load experiments of Chu and James [1993, 1995] on CuAlNi single crystals, it is proved in Ball and James [2002], Ball, Chu and James [2002] (see also Ball, Chu and James [1995]) that certain $\hat{\mathbf{y}}$ with $D \hat{\mathbf{y}}=\mathbf{A}=$ constant are local (but not global) minimizers in $L^{1}\left(\Omega ; \mathbf{R}^{3}\right)$, by an argument exploiting the geometric incompatibility of $\mathbf{A}$ with deformation gradients having lower energy.

How can one prove the existence of equilibrium solutions that are not local minimizers? In exceptional cases one may know an equilibrium solution explicitly (for example a trivial solution) and be able to show that it does not satisfy some necessary condition for a local minimizer. If we can also prove the existence of a global minimizer then we have at least two equilibrium solutions. This can be done, for example, for the case of some mixed boundary-value problems when the stored-energy function is polyconvex but not quasiconvex at the boundary (see Ball and Marsden [1984]). Another approach would be to try to use Morse theory or mountain-pass methods, but it is not clear how to do this so that, for example, appropriate conditions of Palais-Smale type can be verified; for results in an interesting model problem see Zhang [2001].

More generally, one can ask for a description of how the set of equilibrium solutions varies as a function of relevant parameters such as boundary displacements or loads. For the pure traction problem near a stress-free state an interesting study of this type is that of Chillingworth, Marsden and Wan [1982, 1983] and Wan and Marsden [1983].

Problem 10. Develop local and global bifurcation theories for nonlinear elastostatics that apply to mixed displacement-traction boundary conditions.

As an illustration, the most well-known bifurcation problem in elasticity is that of buckling of a thin rod. Although this problem has been treated from the perspective of rod theory in hundreds of papers since the time of Euler [1744], there is no rigorous three-dimensional theory that justifies

Version Dec 16, 2001 ....... Edited by wgm : Typeset on 26 January $2002-9$ h38 
the usual picture of buckling, for example the existence of critical buckling loads or displacements, with corresponding branches of bifurcating buckled solutions. There are at least two difficulties in providing such a theory. The first is that unless the problem is formulated in a somewhat unrealistic way, there is no sufficiently explicit trivial compressed solution about which to linearize the equilibrium equations. For example, suppose that in a stressfree reference configuration a homogeneous isotropic elastic rod occupies the region $\Omega=(0, L) \times D$, where $D \subset \mathbf{R}^{2}$ is the cross-section. A natural boundary-value problem to consider, corresponding to clamped ends, consists of the equilibrium equations (2.4) and the boundary conditions (2.1), (2.6), with the choices $\partial \Omega_{1}=\{0, L\} \times D, \partial \Omega_{2}=(0, L) \times \partial D$, and

$$
\overline{\mathbf{y}}\left(0, \mathbf{x}^{\prime}\right)=\left(0, \mathbf{x}^{\prime}\right), \quad \overline{\mathbf{y}}\left(L, \mathbf{x}^{\prime}\right)=\left(\lambda L, \mathbf{x}^{\prime}\right), \quad \mathbf{x}^{\prime} \in D,
$$

where $\lambda>0$. For $\lambda \neq 1$ the homogeneous deformation $\mathbf{y}\left(x_{1}, \mathbf{x}^{\prime}\right)=\left(\lambda x_{1}, \mathbf{x}^{\prime}\right)$ does not in general satisfy the zero traction condition (2.6). For example, for the compressive case $\lambda<1$ the rod will typically want to bulge, leading to boundary layers near $x_{1}=0$ and $x_{1}=L$. In order to have a homogeneously deformed trivial solution

$$
\mathbf{y}(\mathbf{x})=\left(\lambda x_{1}, \mu x_{2}, \mu x_{3}\right),
$$

one can replace (2.30) by the conditions

$$
y_{1}\left(0, \mathbf{x}^{\prime}\right)=0, \quad y_{1}\left(L, \mathbf{x}^{\prime}\right)=\lambda L, \quad \mathbf{x}^{\prime} \in D,
$$

corresponding to the less realistic case of frictionless end-faces constrained to lie in the planes $\{0\} \times \mathbf{R}^{2}$ and $\{\lambda L\} \times \mathbf{R}^{2}$. To prevent sliding of the end-faces one could add the further constraint that

$$
\int_{D} y_{2} d \mathbf{x}^{\prime}=\int_{D} y_{3} d \mathbf{x}^{\prime}=0 \quad \text { at } x_{1}=0, L .
$$

In this case the natural boundary conditions at $x_{1}=0, L$ for the variational problem are that the stress vector $\mathbf{t}$ across the end-faces has constant transverse components $t_{2}, t_{3}$ which are equal at $x_{1}=0, L$. If we try to prescribe compressive loads at $x_{1}=0, L$ rather than displacements we encounter other difficulties (see Ball [1996a] for a discussion of one of these).

The second more serious difficulty has already been mentioned, namely the lack of regularity of solutions to the linearized equilibrium equations as one approaches points of $\overline{\partial \Omega_{1}} \cap \overline{\partial \Omega_{2}}$, or points of discontinuity of the applied traction in a pure traction formulation of the problem, which prohibits use of the implicit function theorem in natural spaces. Perhaps it might be possible to work in spaces with suitable weights in the neighbourhood of $\overline{\partial \Omega_{1}} \cap \overline{\partial \Omega_{2}}$. But it seems odd that fine details of what goes on near $\overline{\partial \Omega_{1}} \cap \overline{\partial \Omega_{2}}$ should have a significant bearing on the buckling phenomenon, so perhaps there is a different approach to be discovered that circumvents this difficulty.

Version Dec 16, 2001 Edited by wgm : Typeset on 26 January $2002-9$ h 38 
Once a local bifurcation picture has been established, the next thing to understand is what happens to bifurcating solutions for large parameter values. For the case when $\overline{\partial \Omega_{1}} \cap \overline{\partial \Omega_{2}}$ is empty global results have recently been obtained by Healey and Rosakis [1997], Healey and Simpson [1998] and Healey [2000].

\subsection{Energy minimization and fracture.}

Many of the problems described above have generalizations to variational models of fracture. Since typical fracture problems are described by deformations that have jump discontinuities across two-dimensional crack surfaces, fracture cannot in general be modelled in the context of Sobolev spaces. A generalization of the energy functional (2.2) to deformations allowing for fracture is

$$
I(y)=\int_{\Omega} W(D \mathbf{y}) d \mathbf{x}+\int_{S_{\mathbf{y}}} g\left(\mathbf{y}^{+}-\mathbf{y}^{-}, \nu_{\mathbf{y}}\right) d \mathcal{H}^{2},
$$

where $\mathbf{y}$ belongs to the class $\operatorname{SBV}(\Omega)$ of mappings of special bounded variation, i.e., those whose gradient is a bounded measure having no Cantor part. In (2.31) $S_{\mathbf{y}}$ denotes the set of jump points of $\mathbf{y}, \nu_{\mathbf{y}}$ the measure theoretic normal to $S_{\mathbf{y}}$, and $\mathbf{y}^{ \pm}$the traces of $\mathbf{y}$ on either side of $S_{\mathbf{y}}$. The second integral represents the surface energy of cracks, as postulated in the Griffith theory of fracture (see, for example, Cherepanov [1998]), the simplest case $g=$ constant corresponding to a contribution to the energy proportional to the total crack surface area $\mathcal{H}\left(S_{\mathbf{y}}\right)$. Despite much deep work on such models (see, for example, Acerbi, Fonseca and Fusco [1997], Ambrosio [1989, 1990], Ambrosio and Braides [1995], Ambrosio, Fusco and Pallara [1997, 2000], Ambrosio and Pallara [1997], Braides [1998], Braides and Coscia [1993, 1994], Braides, Dal Maso and Garroni [1999], Buttazzo [1995]), and their apparent potential for making an impact on understanding fracture, there have been only isolated attempts to discover their implications for practical problems of fracture mechanics (see, for example, Francfort and Marigo [1998], Bourdin, Francfort and Marigo [2000]).

Problem 11. Clarify the status of models based on the energy functional (2.31) with respect to classical fracture mechanics and to nonlinear elastostatics.

Two key issues are fracture initiation and stability, which are both related to the study of local minimizers for the functional (2.31). A technical obstacle in such a study is the lack of a general method of calculating a general variation of $I$ about a given $\mathbf{y}$ in the direction of nearby deformations having possibly very different sets of jump points. An understanding of local minimizers would also clarify the status of the nonlinear elastostatics model based on (2.2) with respect to that based on (2.31), and thereby 
demystify the apparent sensitivity of the elastostatics model to growth behaviour for very large strains.

\section{Dynamics}

\subsection{Continuum thermomechanics}

We recall briefly the elements of continuum thermomechanics. The basic balance laws are the balance of linear momentum

$$
\frac{d}{d t} \int_{E} \rho_{R} \mathbf{y}_{t} d \mathbf{x}=\int_{\partial E} \mathbf{t}_{R} d S+\int_{E} \mathbf{b} d \mathbf{x},
$$

the balance of angular momentum

$$
\frac{d}{d t} \int_{E} \rho_{R} \mathbf{x} \wedge \mathbf{y}_{t} d \mathbf{x}=\int_{\partial E} \mathbf{x} \wedge \mathbf{t}_{R} d S+\int_{E} \mathbf{x} \wedge \mathbf{b} d \mathbf{x},
$$

and the balance of energy

$$
\begin{aligned}
\frac{d}{d t} \int_{E}\left(\frac{1}{2} \rho_{R}\left|\mathbf{y}_{t}\right|^{2}+U\right) d \mathbf{x}= & \int_{E} \mathbf{b} \cdot \mathbf{y}_{t} d \mathbf{x}+\int_{\partial E} \mathbf{t}_{R} \cdot \mathbf{y}_{t} d S \\
& +\int_{E} r d \mathbf{x}-\int_{\partial E} \mathbf{q}_{R} \cdot \mathbf{n} d S .
\end{aligned}
$$

Here $\mathbf{y}=\mathbf{y}(\mathbf{x}, t)$ denotes the deformation, $\mathbf{t}_{R}$ the Piola-Kirchhoff stress vector, $\rho_{R}>0$ the (constant) density in the reference configuration, $\mathbf{b}$ the body force, $U$ the internal energy, $\mathbf{q}_{R}$ the heat flux vector and $r$ the heat supply. The balance laws are assumed to hold for all Lipschitz domains $E \subset \Omega$, and the unit outward normal to $\partial E$ is denoted by $\mathbf{n}$. In addition to the balance laws, thermomechanical processes are required to obey the Second Law of Thermodynamics, which we assume to hold in the form of the Clausius-Duhem inequality

$$
\frac{d}{d t} \int_{E} \eta d \mathbf{x} \geq-\int_{\partial E} \frac{\mathbf{q}_{R} \cdot \mathbf{n}}{\theta} d S+\int_{E} \frac{r}{\theta} d \mathbf{x}
$$

for all $E$, where $\eta$ is the entropy and $\theta$ the temperature. Standard arguments now show that $\mathbf{t}_{R}=\mathbf{T}_{R} \mathbf{n}$, where $\mathbf{T}_{R}$ is the Piola-Kirchhoff stress tensor, and that for sufficiently smooth processes $(3.1),(3.3),(3.4)$ reduce to the pointwise forms

$$
\begin{aligned}
\rho_{R} \mathbf{y}_{t t}-\operatorname{div} \mathbf{T}_{R}-\mathbf{b} & =0, \\
\frac{d}{d t}\left(\frac{1}{2}\left|\mathbf{y}_{t}\right|^{2}+U\right)-\mathbf{b} \cdot \mathbf{y}_{t}-\operatorname{div}\left(\mathbf{y}_{t} \mathbf{T}_{R}\right)+\operatorname{div} \mathbf{q}_{R}-r & =0 \\
\eta_{t}+\operatorname{div}\left(\frac{\mathbf{q}_{R}}{\theta}\right)-\frac{r}{\theta} & \geq 0,
\end{aligned}
$$

Version Dec 16, 2001 
and that (3.2) is equivalent to the symmetry of the Cauchy stress tensor

$$
\mathbf{T}=(\operatorname{det} D \mathbf{y})^{-1} \mathbf{T}_{R}(D \mathbf{y})^{T}
$$

Eliminating $r$ from (3.6), (3.7), using (3.5) and denoting by

$$
\psi=U-\theta \eta
$$

the Helmholtz free energy, we obtain that for sufficiently smooth processes

$$
-\psi_{t}-\theta_{t} \eta+\mathbf{T}_{R} \cdot D \mathbf{y}_{t}-\frac{\mathbf{q}_{R} \cdot \operatorname{grad} \theta}{\theta} \geq 0
$$

Adopting the prescription of Coleman and Noll [1963], we assume that given an arbitrary deformation $\mathbf{y}=\mathbf{y}(\mathbf{x}, t)$ and temperature field $\theta=\theta(\mathbf{x}, t)$ we can choose a body force $\mathbf{b}=\mathbf{b}(\mathbf{x}, t)$ and heat supply $r=r(\mathbf{x}, t)$ to balance (3.5), (3.6), so that (3.9) becomes an identity to be satisfied by the constitutive equations. For the case of a thermoelastic material, for which $T_{R}, \eta, \psi, \mathbf{q}_{R}$ are assumed to be functions of $D \mathbf{y}, \theta, \operatorname{grad} \theta$, this leads to the relations

$$
\psi=\psi(D \mathbf{y}, \theta), \quad \mathbf{T}_{R}=D_{\mathbf{A}} \psi, \quad \eta=-D_{\theta} \psi,
$$

and then (3.9) reduces to the inequality

$$
-\frac{\mathbf{q}_{R} \cdot \operatorname{grad} \theta}{\theta} \geq 0
$$

(Note that, although this inequality must be satisfied by the constitutive equation for $\mathbf{q}_{R}$, for processes involving shocks (3.11) is not equivalent to (3.7), since the cancellations in the argument used to obtain (3.9) are no longer valid.) For thermoelastic materials the balance of angular momentum is satisfied identically as a consequence of the requirement that $\mathbf{T}_{R}$ is frame-indifferent, i.e.

$$
\mathbf{T}_{R}(\mathbf{R A}, \theta)=\mathbf{R T}_{R}(\mathbf{A}, \theta), \quad \text { for all } \mathbf{R} \in \mathrm{SO}(3),
$$

which is equivalent to the condition that

$$
\psi(\mathbf{R A}, \theta)=\psi(\mathbf{A}, \theta), \quad \text { for all } \mathbf{R} \in \mathrm{SO}(3) .
$$

The condition of material symmetry becomes

$$
\psi(\mathbf{A Q}, \theta)=\psi(\mathbf{A}, \theta), \quad \text { for all } \mathbf{Q} \in \mathcal{S}
$$

where $\mathcal{S}$ is the isotropy group. The equations of isothermal thermoelasticity are obtained from (3.5), (3.10) by assuming that $\theta(\mathbf{x}, t)=\theta_{0}=$ constant. Thus the balance of linear momentum becomes

$$
\rho_{R} \mathbf{y}_{t t}-\operatorname{div} D_{\mathbf{A}} W(D \mathbf{y})-\mathbf{b}=0,
$$

Version Dec 16, 2001 Edited by wgm : Typeset on 26 January $2002-9$ h 38 
where $W(\mathbf{A})=\psi\left(\mathbf{A}, \theta_{0}\right)$. As regards the entropy inequality, we again adopt the Coleman and Noll point of view, choosing $r$ to balance (3.6). (Here we follow Dafermos [2000], who gives a similar reduction for isentropic thermoelasticity.) Since, from (3.11), $\mathbf{q}_{R}=0$ when $\operatorname{grad} \theta=0,(3.7)$ becomes

$$
\left(\frac{1}{2} \rho_{R}\left|\mathbf{y}_{t}\right|^{2}+\psi\right)_{t}-\mathbf{b} \cdot \mathbf{y}_{t}-\operatorname{div}\left(\mathbf{y}_{t} \mathbf{T}_{R}\right) \leq 0 .
$$

For the more general case of a thermoviscoelastic material (of strain-rate type), $T_{R}, \eta, \psi, \mathbf{q}_{R}$ are assumed to be functions of $D \mathbf{y}, D \mathbf{y}_{t}, \theta, \operatorname{grad} \theta$. By the same method we find that

$$
\psi=\psi(D \mathbf{y}, \theta), \quad \eta=-D_{\theta} \psi
$$

and that

$$
\mathbf{S} \cdot D \mathbf{y}_{t}-\frac{\mathbf{q}_{R} \cdot \operatorname{grad} \theta}{\theta} \geq 0
$$

where

$$
\mathbf{T}_{R}=D_{\mathbf{A}} \psi+\mathbf{S}\left(D \mathbf{y}, D \mathbf{y}_{t}, \theta, \operatorname{grad} \theta\right) .
$$

In the isothermal case we obtain the equation of motion

$$
\rho_{R} \mathbf{y}_{t t}-\operatorname{div} D_{\mathbf{A}} W(D \mathbf{y})-\operatorname{div} \mathbf{S}\left(D \mathbf{y}, D \mathbf{y}_{t}\right)-\mathbf{b}=0,
$$

where $W=\psi\left(D \mathbf{y}, \theta_{0}\right), \mathbf{S}\left(D \mathbf{y}, D \mathbf{y}_{t}\right)=\mathbf{S}\left(D \mathbf{y}, D \mathbf{y}_{t}, \theta_{0}, 0\right)$, together with the energy inequality (3.15). The frame-indifference of $\mathbf{S}$ takes the form

$$
\mathbf{S}\left(D \mathbf{y}, D \mathbf{y}_{t}\right)=D \mathbf{y} \Sigma\left(\mathbf{U}, \mathbf{U}_{t}\right),
$$

for some matrix-valued function $\Sigma$, where $\mathbf{U}=\left(D \mathbf{y}^{T} D \mathbf{y}\right)^{1 / 2}$.

\subsection{Existence of solutions}

Problem 12. Prove the global existence and uniqueness of solutions to initial boundary-value problems for properly formulated dynamic theories of nonlinear elasticity.

To discuss this problem let us begin with isothermal thermoelasticity. The governing equations are (3.14). These equations need to be supplemented by boundary conditions such as (2.1), (2.6) and by the initial conditions

$$
\mathbf{y}(\mathbf{x}, 0)=\mathbf{y}_{0}(\mathbf{x}), \quad \mathbf{y}_{t}(\mathbf{x}, 0)=\mathbf{y}_{1}(\mathbf{x}) .
$$

If the body force is conservative, so that

$$
\mathbf{b}=-\operatorname{grad}_{\mathbf{y}} h(\mathbf{x}, \mathbf{y}),
$$

Version Dec 16, 2001

. Edited by wgm : Typeset on 26 January $2002-9$ h 38 
then (3.14) formally comprises a Hamiltonian system, and could be alternatively obtained by applying Hamilton's principle to the functional

$$
\int_{0}^{T} \int_{\Omega}\left(\frac{1}{2} \rho_{R}\left|\mathbf{y}_{t}\right|^{2}-W(D \mathbf{y})-h(\mathbf{x}, \mathbf{y})\right) d \mathbf{x} d t .
$$

In particular, solutions formally satisfy the balance of energy

$$
E\left(\mathbf{y}, \mathbf{y}_{t}\right)=E\left(\mathbf{y}_{0}, \mathbf{y}_{1}\right), \quad t \geq 0
$$

where

$$
E(\mathbf{y}, \mathbf{v})=\int_{\Omega}\left(\frac{1}{2} \rho_{R}|\mathbf{v}|^{2}+W(D \mathbf{y})+h(\mathbf{x}, \mathbf{y})\right) d \mathbf{x} .
$$

However, weak solutions of the quasilinear wave equation (3.14) do not in general satisfy (3.20), since singularities such as shock waves can dissipate energy. Correspondingly, although equality holds in the dissipation inequality (3.15) for smooth solutions, in general it does not do so for weak solutions. Interpreted in the sense of distributions or measures, (3.15) acts as an admissibility criterion for weak solutions.

In one dimension (3.14) takes the form

$$
\rho_{R} y_{t t}-\sigma\left(y_{x}\right)_{x}-b=0,
$$

where $\sigma\left(y_{x}\right)=W^{\prime}\left(y_{x}\right)$, which setting $u_{1}=\rho_{R} y_{t}, u_{2}=y_{x}$ is equivalent to the system of two conservation laws

$$
\mathbf{u}_{t}-\mathbf{f}(\mathbf{u})_{x}=\mathbf{g}
$$

where

$$
\mathbf{f}(\mathbf{u})=\left(\begin{array}{c}
\sigma\left(u_{2}\right) \\
\rho_{R}^{-1} u_{1}
\end{array}\right), \quad \mathbf{g}=\left(\begin{array}{l}
b \\
0
\end{array}\right) .
$$

This system is strictly hyperbolic if $\sigma^{\prime}=W^{\prime \prime}>0$, so that $W$ is strictly convex.

Two approaches have been employed to study (3.22), the Glimm scheme, Glimm [1965], and variants of it such as front-tracking (introduced by Dafermos [1972]), and the method of compensated compactness as pioneered by Tartar [1979, 1982] and DiPerna [1983, 1985].

The Glimm scheme and variants apply to strictly hyperbolic systems of the form (3.22) with $\mathbf{u} \in \mathbf{R}^{n}, \mathbf{f}: \mathbf{R}^{n} \rightarrow \mathbf{R}^{n}, \mathbf{g} \in \mathbf{R}^{n}$. They involve a semi-explicit construction of the solutions in terms of approximation of the initial data by piecewise constant functions, together with an analysis of wave interactions. They are restricted to initial data having small total variation, and thus, via total variation estimates on the solution, to solutions of small total variation. Glimm's original work assumed that the system 
was 'genuinely nonlinear', but this restriction was removed by Liu [1981]. Thanks to work of Bressan [1988, 1995], Bressan and Colombo [1995], Bressan [Crasta and Piccoli], Bressan and Goatin [1999], Bressan and Le Floch [1997], Bressan and Lewicka [2000], Bressan, Liu and Yang [1999] and Liu and Yang $[1999 \mathrm{~b}, \mathrm{a}, \mathrm{c}]$, the solutions obtained in these ways are now known to be unique in appropriate function classes. For genuinely nonlinear systems of two conservation laws, such as (3.21) with $W^{\prime \prime}>0, W^{\prime \prime \prime} \neq 0$, more is known (see [Dafermos, 2000, Chapter XI]). Most of this work is for solutions on the whole real line; for a treatment of (3.21) with displacement boundary conditions see Liu [1977].

The method of compensated compactness, on the other hand, has up to now been restricted to systems of at most two conservation laws, such as (3.21). Starting from a sequence of approximate solutions obtained from the method of vanishing viscosity (or by a variational time-discretization scheme, see Demoulini, Stuart and Tzavaras [2000]), it uses information coming from the existence of a suitable family of 'entropies' (quantities for which there is a corresponding conservation law satisfied by smooth solutions) to pass to the limit using weak convergence. However, there is no corresponding uniqueness theorem. These results are described in the books of Bressan [2000], Dafermos [2000] and Serre [2000]. In a recent development, Bianchini and Bressan [2001] have made a breakthrough by obtaining for the first time total variation estimates directly from the vanishing viscosity method.

For the three-dimensional equations (3.14) very little is known. Hughes, Kato and Marsden [1977] proved that if $W$ satisfies the strong ellipticity condition

$$
D^{2} W(\mathbf{A})(\mathbf{a} \otimes \mathbf{n}, \mathbf{a} \otimes \mathbf{n}) \geq \mu|\mathbf{a}|^{2}|\mathbf{n}|^{2}
$$

for all $\mathbf{A} \in M_{+}^{3 \times 3}, \mathbf{a}, \mathbf{n} \in \mathbf{R}^{3}$, where $\mu>0$, then for smooth initial data (3.18) defined on the whole of $\mathbf{R}^{3}$ with $\operatorname{det} D \mathbf{y}_{0}>0$, there exists a unique smooth solution on a small time interval $[0, T), T>0$. This result was extended to pure displacement boundary conditions by Kato [1985]. For related results see Dafermos and Hrusa [1985] and [Dafermos, 2000, Chapter V]. There seem to be no short-time existence results known for mixed displacement-traction boundary conditions. Interesting results concerning large time existence for sufficiently smooth and small initial data on the whole of $R^{3}$ have been obtained by John [1988]. For corresponding results for incompressible elasticity see Hrusa and Renardy [1988], Ebin and Saxton [1986], Ebin and Simanca [1990, 1992] and Ebin [1993, 1996].

In the variables $\mathbf{A}=D \mathbf{y}, \mathbf{p}=\rho_{R} \mathbf{y}_{t},(3.14)$ becomes the system

$$
\begin{aligned}
& \mathbf{A}_{t}=\mathbf{D}, \quad D_{i j}=\rho_{R}^{-1} v_{i, j}, \\
& \mathbf{p}_{t}=\operatorname{div} D_{\mathbf{A}} W(D \mathbf{y})+\mathbf{b},
\end{aligned}
$$

Version Dec 16, 2001 ....... Edited by wgm : Typeset on 26 January $2002-9$ h38 
which is hyperbolic if

$$
D^{2} W(\mathbf{A})(\mathbf{a} \otimes \mathbf{n}, \mathbf{a} \otimes \mathbf{n})>0 \quad \text { for all } \mathbf{A} \in M_{+}^{3 \times 3} \text { and nonzero } \mathbf{a}, \mathbf{n} \in \mathbf{R}^{3} .
$$

There is no theory of weak solutions for such multi-dimensional systems. In particular, it is unclear what conditions on $W$ are natural for existence, and whether these conditions will be the same as those guaranteeing existence for elastostatics, namely quasiconvexity or polyconvexity. The system (3.24), (3.25) is special in the sense that there is an involution

$$
A_{i j, k}-A_{i k, j}=0
$$

which is satisfied by all weak solutions. Exploiting this in the context of a general system having involutions, Dafermos [1996, 2000] proves a theorem implying that if $W$ is quasiconvex and satisfies (3.23) then any Lipschitz solution $\mathbf{A}, \mathbf{p}$ of $(3.24),(3.25)$ on $\mathbf{R}^{3} \times[0, T], T>0$, is unique within the class of weak solutions admissible with respect to the entropy $W$, of uniformly small local oscillation, and satisfying the same initial data as A, p. An unpublished idea of LeFloch, found independently by Qin [1998], leads to the observation that for polyconvex $W$ the hypothesis of uniformly small oscillation can be removed. These results are interesting because they so far represent the only use of quasiconvexity and polyconvexity in the context of dynamics. See Šverák [1995] for an idea of how quasiconvexity (in an augmented space) might be used to prove existence by passage to the limit using weak convergence, in the spirit of compensated compactness. For the full system of three-dimensional nonlinear thermoelasticity (3.5)-(3.7), which has the additional conservation law (3.6), the state of knowledge (or rather lack of it) is similar. For these systems an additional difficulty is that of ensuring invertibility of solutions, and in particular the condition $\operatorname{det} D \mathbf{y}(\mathbf{x}, t)>0$.

For a thermoviscoelastic material, one can hope that a sufficiently wellbehaved viscous part $\mathbf{S}$ of the stress will guarantee existence without any convexity conditions on $W$. Indeed, in the one-dimensional isothermal case, for which the equation of motion takes the form

$$
\rho_{R} y_{t t}-\sigma\left(y_{x}, y_{x t}\right)_{x}-b=0,
$$

for which Dafermos [1969] and Antman and Seidman [1996] have proved existence and uniqueness for a general class of $\sigma$. The special case of the equation

$$
\rho_{R} y_{t t}-\sigma\left(y_{x}\right)_{x}-y_{x x t}=0 \text {. }
$$

has been treated in numerous papers (see, for example, Greenberg, MacCamy and Mizel [1967], Andrews [1980], Pego [1987]). For corresponding results for thermoviscoelasticity see Racke and Zheng [1997].

Version Dec 16, 2001 ....... Edited by wgm : Typeset on 26 January $2002-9$ h38 
For the isothermal case in three dimensions it is natural to consider in place of (3.27) the equation

$$
\rho_{R} \mathbf{y}_{t t}-\operatorname{div} D_{\mathbf{A}} W(D \mathbf{y})-\Delta \mathbf{y}_{t}=0,
$$

for which a theory of existence is available (see Rybka [1992], Friesecke and Dolzmann [1997]). However the corresponding viscous stress $\mathbf{S}=D \mathbf{y}_{t}$ is not of the form (3.17), and so is not frame-indifferent. The only existence theory for weak or strong solutions of (3.16) with $\mathbf{S}$ frame-indifferent appears to be that of Potier-Ferry [1981, 1982], who, for pure displacement boundary conditions, established global existence and uniqueness of solutions for initial data close to a smooth equilibrium having strictly positive second variation. Potier-Ferry assumed that the linearized elasticity operator at the equilibrium is strongly elliptic, and that a corresponding positivity condition holds for the linearized viscous stress. He uses methods of Sobolevskii [1966] (for an alternative treatment also based on Sobolevskii's work see Xu and Marsden [1996] and Xu [2000]).

A recent monograph covering various aspects of the analysis of thermoelasticity is that of Jiang and Racke [2000].

A different approach to the existence of solutions in elasticity is to weaken the concept of solution to that of a measure-valued solution, in which the unknown is a Young measure $\nu_{\mathbf{x}, t}$ in appropriate variables satisfying an integral identity obtained by formally passing to the weak limit in a sequence of approximate solutions. Using a variational time-discretization method, the global existence of such solutions has been proved by Demoulini [2000] for the viscoelastic equation (3.16) with $\mathbf{S}$ frame-indifferent, and by Demoulini, Stuart and Tzavaras [2001] for the equations (3.14) of elastodynamics with $W$ polyconvex (exploiting the idea of Le Floch and Qin [1998]). However they are unable to handle the constraint $\operatorname{det} D \mathbf{y}>0$. Of course the significance of such results would be greatly enhanced if there were examples known of cases in which there was no corresponding weak solution.

\subsection{The relation between statics and dynamics}

For suitable boundary conditions, the Second Law of Thermodynamics endows the equations of motion of continuum thermodynamics with a Lyapunov function, that is a function of the state variables that is nonincreasing along solutions. For example, suppose that the mechanical boundary conditions are that $\mathbf{y}=\mathbf{y}(\mathbf{x}, t)$ satisfies

$$
\left.\mathbf{y}(\cdot, t)\right|_{\partial \Omega_{1}}=\overline{\mathbf{y}}(\cdot)
$$

and the condition that the applied traction on $\partial \Omega_{2}$ is zero, and that the thermal boundary condition is

$$
\left.\theta(\cdot, t)\right|_{\partial \Omega}=\theta_{0},
$$

Version Dec 16, 2001 ....... Edited by wgm : Typeset on 26 January $2002-9$ h38 
where $\theta_{0}>0$ is a constant. Assume that the heat supply $r$ is zero, and that the body force is given by (3.19). Then from (3.1), (3.3) and (3.4) with $E=\Omega$ we find that the ballistic free energy

$$
\mathcal{E}=\int_{\Omega}\left[\frac{1}{2} \rho_{R}\left|\mathbf{y}_{t}\right|^{2}+U-\theta_{0} \eta+h\right] d \mathbf{x}
$$

is nonincreasing along solutions. (This is a result of Duhem [1911] for the case of thermoelasticity. See Coleman and Dill [1973], Ericksen [1966] and Ball [1986, 1992] for further discussion and references.) For a thermoviscoelastic material, if $\mathbf{v}(\cdot, t) \rightarrow 0, \mathbf{y}(\cdot, t) \rightarrow \mathbf{y}(\cdot)$ and $\theta(\cdot, t) \rightarrow \theta_{0}$ as $t \rightarrow \infty$ the integrand in (3.28) formally tends to $W(D \mathbf{y})+h(\mathbf{x}, \mathbf{y})$, where $W(D \mathbf{y})=\psi\left(D \mathbf{y}, \theta_{0}\right)$ and $\psi$ is the Helmholtz free energy

$$
\psi\left(D \mathbf{y}, \theta_{0}\right)=U\left(D \mathbf{y}, \theta_{0}\right)-\theta_{0} \eta\left(D \mathbf{y}, \theta_{0}\right) .
$$

This motivates minimization of

$$
I(\mathbf{y})=\int_{\Omega}[W(D \mathbf{y})+h(\mathbf{x}, \mathbf{y})] d \mathbf{x} .
$$

(For pure zero traction boundary conditions, when uniformly rotating equilibria are to be expected, we do not expect that $\mathbf{y}_{t} \rightarrow 0$, and the corresponding entropy maximization problem is studied by Lin [1990]). As applied to such problems, the calculus of variations can be viewed as representing a crude version of dynamics in which true dynamic orbits are replaced by all paths in a phase space of mappings $\left\{\mathbf{y}, \mathbf{y}_{t}, \theta\right\}$ along which $\mathcal{E}$ is nonincreasing.

Problem 13. Develop a qualitative dynamics for dynamic theories of elasticity.

Of course a prerequisite for such a qualitative dynamics is a global existence theory for solutions. Given such a theory, the points at issue are the usual ones for dissipative dynamical systems, namely whether solutions converge to equilibrium states as $t \rightarrow \infty$, the structure of regions of attraction, the existence of stable and unstable manifolds of equilibria, the existence of a global attractor, and so on. In particular one can ask whether dynamic orbits generically realize suitably defined local minimizing sequences for the ballistic free energy. This is especially interesting in the case when the ballistic free energy does not attain a minimum (as in models of elastic crystals - see Section 4.2). In fact for the one-dimensional viscoelastic model of this type studied by Ball, Holmes, James, Pego, and Swart [1991] and Friesecke and McLeod [1996], it is known that no dynamic solutions realize global minimizing sequences; it is unclear whether or not this is a one-dimensional phenomenon.

Problem 14. Develop criteria for the dynamic stability and instability of equilibria.

Version Dec 16, $2001 \ldots \ldots$. Edited by wgm : Typeset on 26 January $2002-9$ h38 
Koiter [1976] is among those who have drawn attention to the problem of justifying the energy criterion for stability, that an equilibrium solution is stable if it is a local minimizer of the corresponding elastic energy (for example, of the ballistic free energy for a thermoviscoelastic material). To keep the discussion simple, consider the case of isothermal motion of a thermoviscoelastic material, for which the equation of motion is given by (3.16), and assume that the body force is zero. The corresponding Lyapunov function is

$$
E(\mathbf{y}, \mathbf{v})=\int_{\Omega}\left[\frac{1}{2} \rho_{R}|\mathbf{v}|^{2}+W(D \mathbf{y})\right] d \mathbf{x},
$$

for which $\mathbf{y}=\mathbf{y}^{*}, \mathbf{v}=0$ is a local minimizer provided $\mathbf{y}^{*}$ is a local minimizer of

$$
E(\mathbf{y})=\int_{\Omega} W(D \mathbf{y}) d \mathbf{x} .
$$

Since there are different types of local minimizer corresponding to different metrics $d$ on different spaces $X$ of deformations $\mathbf{y}$, in particular $W^{1, \infty}$ local minimizers and $W^{1, p}$ local minimizers for $1 \leq p<\infty$, it is not clear which kinds of local minimizers are needed to ensure dynamical stability. As emphasised, for example, by Knops and Wilkes [1973], the standard argument for establishing Lyapunov stability with respect to a metric $d$ requires more than just that $\mathbf{y}^{*}$ is a strict local minimizer with respect to $d$ (that is $I(\mathbf{y})>I\left(\mathbf{y}^{*}\right)$ whenever $\mathbf{y} \neq \mathbf{y}^{*}$ and $d\left(\mathbf{y}, \mathbf{y}^{*}\right)$ is sufficiently small). What is needed, in addition to the continuity of $I$ with respect to $d$ and the continuity in time of dynamic orbits with respect to $d$, is that $\mathbf{y}^{*}$ lies in a potential well, namely that for some $\varepsilon>0$

$$
\inf _{d\left(\mathbf{y}, \mathbf{y}^{*}\right)=\varepsilon} I(\mathbf{y})>I\left(\mathbf{y}^{*}\right) .
$$

For a way of verifying that a strict local minimizer in a space based on $W^{1, p}$ for $1<p<\infty$ satisfies this requirement when $W$ is strictly polyconvex see Ball and Marsden [1984], and for the case when $W$ is strictly quasiconvex see Evans and Gariepy [1987] and Sychev [1999]. However, we do not know in general how to prove that a given $\mathbf{y}^{*}$ is a strict $W^{1, p}$ local minimizer. Further, for $W$ that are not quasiconvex almost nothing is known. These questions are related to the open problem, already mentioned in Section 2.7, of generalizing to higher dimensions the Weierstrass fundamental sufficiency theorem.

The only rigorous result justifying the energy criterion in any generality (in particular, in three dimensions) seems to be that of Potier-Ferry [1982], who for pure displacement boundary conditions establishes asymptotic stability, with respect to the norm of $W^{2, p} \times W^{2, p}, p>3$, of smooth equilibria having strictly positive second variation, under the hypotheses described in Section 3.2. 
Finally, little is known about criteria justifying instability of an equilibrium solution. An instructive example is that of Friesecke and McLeod [1997], who for a problem of one-dimensional viscoelasticity exhibit an equilibrium solution $\bar{u}$ which, with respect to a topology in which the dynamics is well-posed, (a) is dynamically stable, but (b) is such that there is a continuous path in the phase space (not a dynamical solution) leaving $\bar{u}$ along which the energy strictly decreases, so that in particular $\bar{u}$ is not a local minimizer.

\section{Multiscale problems}

\subsection{From atomic to continuum}

Problem 15. Establish the status of elasticity theory with respect to atomistic models.

Is it possible to derive elasticity theory from atomistic models? Such models range from full quantum many-body theory to approximations such as density-functional theory, Thomas-Fermi theory, and models in which electronic effects are not explicitly considered but incorporated into interatomic potentials. There is an extensive physics and materials literature on such models and on methods for bridging the atomistic and continuum lengthscales (see Phillips [2001] for an introduction). Here I will concentrate on what little is known rigorously for the case of elastic crystals. However, another important class of materials is that of cross-linked polymers, which involves some different issues that are considered from the point of view of statistical mechanics in Deam and Edwards [1976], Edwards and Vilgis [1988].

For crystals, the first question to answer is why they occur in the first place, that is why at low temperature the minimum energy configuration of a very large number of atoms is spatially periodic. This is the famous unsolved 'crystal problem', nicely reviewed by Radin [1987]. Likewise, there is no fundamental understanding of the statistical mechanics of crystals, which would explain their stability and instabilities. Given this impasse, in attempts to pass from atomistic to continuum models of crystals some initial atomic order is always assumed. In the context of free-energy minimization, one can draw a distinction between two kinds of approach. In the first, an appropriate limit of a discrete energy functional is sought along sequences of explicit atomic configurations. For example the atoms may be assumed to occupy a periodic lattice in a reference configuration, and to be displaced according to a given sufficiently smooth continuum deformation $\mathbf{y}$ (the Cauchy-Born hypothesis), the number of atoms being sent to infinity with a suitable scaling for the energy. In this approach there is no attempt to explain why the atoms adopt the assumed configurations. A

Version Dec 16, 2001 ....... Edited by wgm : Typeset on 26 January $2002-9$ h38 
recent example is the work of Blanc, Le Bris and Lions [2001], who obtain in a suitable scaling a limiting energy of the form

$$
I(\mathbf{y})=\int_{\Omega} W(D \mathbf{y}) d x
$$

in the cases of (a) a two-body interaction, (b) Thomas-Fermi theory. As is well-known the case of two-body interactions leads to a $W$ satisfying the Cauchy relations, namely that the linearized elasticity coefficients at a natural state ( say $D \mathbf{y}=\mathbf{1}$ )

$$
c_{i j k l}=\frac{\partial^{2} W(\mathbf{1})}{\partial A_{i j} \partial A_{k l}}
$$

possess the symmetries

$$
c_{i j k l}=c_{j i k l}=c_{k l i j}=c_{i k j l} .
$$

These symmetries are known not to hold in general (see Love [1927], Weiner [1983]). Blanc, LeBris, and Lions also obtain second-order terms in the expansion of the energy with respect to the scaling parameter, identifying these with surface energies. For fundamental results on linear deformations with the Cauchy-Born hypothesis see Lieb and Simon [1977] and Fefferman [1985]; in these papers the dependence on the deformation gradient enters implicitly through the given Bravais lattice. For more recent extensions of the results of Lieb and Simon [1977] see Catto, Le Bris and Lions [1998].

The second approach is to consider the true minimizers of the discrete problems, and to try to understand what functional their limit minimizes. One example of this approach is the interesting study by Friesecke and Theil [2001] of a model two-dimensional problem of a lattice of particles linked by harmonic springs between their nearest and next nearest neighbours. They determine open regions of atomic parameters in which the Cauchy-Born hypothesis holds in the appropriate limit, and open regions in which it does not. Another interesting recent study in this spirit is that of Penrose [2001], who considers a two-dimensional model problem of a lattice of rotatable disks with one-body wall interactions and angle-dependent two-body interactions. By suitably restricting the statistical ensemble, so that, for example, certain angles between atoms in the deformed configuration are constrained to lie in certain intervals (these constraints being designed to deter dislocations), he proves the existence of an elastic free energy $W$ corresponding to taking the thermodynamic limit with prescribed linear boundary data. He also deduces a convexity property of $W$ weaker than rank-one convexity, and suggests that $W$ might in fact be quasiconvex. Other work in this spirit is that of Braides, Dal Maso and Garroni [1999], Braides and Gelli [2001a,b] and Foccardi and Gelli [2001], who calculate the $\Gamma$-limits of certain discrete functionals with nearest-neighbour 
or pairwise interactions, obtaining a limiting functional allowing fracture of the general form (2.31) together with a corresponding function space on which to minimize it (namely $\operatorname{SBV}(\Omega)$ or $\operatorname{GSBV}(\Omega)$ ). This acts as a reminder that, since the predictions of minimization problems can depend on the function space, as we have seen in Section 2.3 in connection with the Lavrentiev phenomenon, a proper atomistic to continuum derivation should deliver not only the limiting governing equations or energy, but the appropriate function space as well.

For a proposed scheme for the passage from atomistic to continuum models for thin films, rods or tubes see Friesecke and James [2000]. There seems to be no work on atomistic derivations of dynamic theories of elasticity, or even of elastostatics in the context of deformations that are not global energy minimizers.

In some situations it is desirable to simultaneously use a continuum and a discrete model. For example, one may wish to study the interaction of a defect or other localized region (such as the vicinity of a crack tip), in which atomistic effects may be important, with the surrounding bulk material, where a continuum theory is appropriate. One way of handling the resulting matching problem is the quasicontinuum method (see Tadmor, Ortiz and Phillips [1996]). A rigorous understanding of such methods is lacking.

\subsection{From microscales to macroscales}

Materials undergoing phase transformations involving a change of shape at a critical temperature typically develop characteristic patterns of $m i$ crostructure, in which the deformation gradient has large variations on a fine length-scale that varies from material to material but can be as small as a few atomic spacings. Such microstructures often contain twinned regions consisting of many parallel layers separated by sharp interfaces, the deformation gradient alternating between two distinct values in adjacent layers.

Why does fine microstructure form? To what extent can we predict its morphology? What are the properties of the material at a macroscale much larger than the microscale of the microstructure? Whereas it would be desirable to answer such questions in the context of a suitably formulated dynamical theory, it is neither clear what such a theory should be (especially as regards the kinetics of interfaces), nor do we currently have the techniques to give answers corresponding to any such theory. Hence we will discuss some more specific open problems that arise in static models of such phase transformations.

Consider a single crystal of thermoelastic material that undergoes a diffusionless phase transformation involving a change of shape at the temperature $\theta_{c}$. For definiteness, suppose that there is an interval $E$ of temperatures, containing $\theta_{c}$, such that for $\theta \in E, \theta>\theta_{c}$, the minimum energy 
configuration (called austenite) of the crystal is cubic. Taking the reference configuration to be undistorted austenite at $\theta=\theta_{c}$, we suppose that for $\theta \in E, \theta \leq \theta_{c}$, a minimum energy configuration (called martensite) is given by the transformation strain $U(\theta)$. We suppose that the Helmholtz freeenergy function $\psi(\mathbf{A}, \theta)$ attains a finite minimum with respect to $\mathbf{A} \in M_{+}^{3 \times 3}$ for each $\theta \in E$, so that by adding to it a suitable function of $\theta$ we may assume for the purposes of free-energy minimization that

$$
\min _{\mathbf{A} \in M_{+}^{3 \times 3}} \psi(\mathbf{A}, \theta)=0 .
$$

Let

$$
K(\theta)=\left\{\mathbf{A} \in M_{+}^{3 \times 3}: \psi(\mathbf{A}, \theta)=0\right\}
$$

be the set of energy-minimizing deformation gradients. Note that by (3.12) and (3.13)

$$
\mathrm{SO}(3) K(\theta) \mathcal{S}=K(\theta)
$$

where we take $\mathcal{S}$ to be the subgroup $P^{24}$ of $\mathrm{SO}(3)$ consisting of the 24 rotations mapping a cube into itself. It is thus reasonable to suppose that for $\theta \in E$,

$$
K(\theta)= \begin{cases}\alpha(\theta) \mathrm{SO}(3), & \theta>\theta_{c}, \\ \mathrm{SO}(3) \cup \bigcup_{i=1}^{N} \mathrm{SO}(3) U_{i}\left(\theta_{c}\right), & \theta=\theta_{c}, \\ \bigcup_{i=1}^{N} \mathrm{SO}(3) U_{i}(\theta), & \theta<\theta_{c}\end{cases}
$$

where $\alpha(\cdot)$ describes the thermal expansion, with $\alpha\left(\theta_{c}\right)=1$, and where the $U_{i}(\theta), 1 \leq i \leq N$, are the distinct positive definite symmetric matrices of the form $Q^{T} U(\theta) Q, Q \in P^{24}$. We assume that $N$ is independent of $\theta \in E$. If $U_{i}\left(\theta_{c}\right) \neq \mathbf{1}$ the transformation is first-order, while if $U_{i}\left(\theta_{c}\right)=\mathbf{1}$ it is secondorder. We say that each $U_{i}$ describes a different variant of martensite. For example, in the case of a cubic-to-tetragonal transformation we may take

$$
U(\theta)=U_{1}(\theta)=\operatorname{diag}\left(\eta_{3}, \eta_{1}, \eta_{1}\right),
$$

where $\eta_{1}(\theta)>0, \eta_{3}(\theta)>0$, and then we find that $N=3$, and that

$$
U_{2}(\theta)=\operatorname{diag}\left(\eta_{1}, \eta_{3}, \eta_{1}\right), \quad U_{3}(\theta)=\operatorname{diag}\left(\eta_{1}, \eta_{1}, \eta_{3}\right) .
$$

For other transformations we get different numbers of variants; for example, for cubic-to-orthorhombic transformations $N=6$, and for cubic to monoclinic transformations $N=12$.

Note that in adopting (4.1) we exclude large shears leaving the crystal lattice invariant (see Ericksen [1977b]), the inclusion of which would lead to $K(\theta)$ consisting of an infinite number of energy wells for each $\theta$, and to 
an energy-minimization problem of a different character to that based on (4.1) (see Fonseca [1988]).

The total free energy corresponding to the deformation $\mathbf{y}: \Omega \rightarrow \mathbf{R}^{3}$ is given by

$$
I_{\theta}(\mathbf{y})=\int_{\Omega} \psi(D \mathbf{y}, \theta) d \mathbf{x}
$$

Zero-energy microstructures (at the temperature $\theta$ ) correspond to sequences of deformations $\mathbf{y}^{(j)}$ such that

$$
\lim _{j \rightarrow \infty} I_{\theta}\left(\mathbf{y}^{(j)}\right)=0
$$

If we assume a mild growth condition on $\psi$, such as

$$
\psi(\mathbf{A}, \theta) \geq c_{0}|\mathbf{A}|^{p}-c_{1} \quad \text { for all } \mathbf{A} \in M_{+}^{3 \times 3},
$$

where $c_{0}>0, c_{1}$ and $p>1$ are constants, then (4.2) is equivalent to the statement that $D \mathbf{y}^{(j)} \rightarrow K(\theta)$ in measure, or that the Young measure $\left(\nu_{\mathbf{x}}\right)_{\mathbf{x} \in \Omega}$ corresponding to (a subsequence of) $D \mathbf{y}^{(j)}$ satisfies

$$
\operatorname{supp} \nu_{x} \subset K(\theta) \quad \text { a.e. } \mathbf{x} \in \Omega \text {. }
$$

The set of macroscopic deformation gradients corresponding to zero-energy microstructures is the set of gradients $D \mathbf{y}: \Omega \rightarrow M_{+}^{3 \times 3}$ such that $D \mathbf{y}^{(j)} \rightarrow$ $D \mathbf{y}$ in $L^{p}$ for some sequence $y^{(j)}$ satisfying (4.2). Equivalently, following the results of Kinderlehrer and Pedregal [1991, 1994], this is the set of gradients $D$ y such that

$$
D \mathbf{y}(\mathbf{x}) \in K(\theta)^{\mathrm{qc}} \quad \text { a.e. } \mathbf{x} \in \Omega \text {, }
$$

where for a compact set $K \subset M^{3 \times 3}, K^{\mathrm{qc}}$ denotes the quasiconvexification of $K$. Equivalent definitions of $K^{\mathrm{qc}}$ are

$$
\begin{aligned}
K^{\mathrm{qc}} & =\left\{\bar{\nu}: \nu \text { is a homogeneous } W^{1, \infty} \text { Young measure with supp } \nu \subset K\right\} \\
& =\left\{\mathbf{A} \in M^{3 \times 3}: \varphi(\mathbf{A}) \leq \max _{\mathbf{B} \in K} \varphi(\mathbf{B}) \text { for all quasiconvex } \varphi\right\} \\
& =\bigcap\{E \supset K: E \text { quasiconvex }\} .
\end{aligned}
$$

Here $\mathcal{A}_{\mathbf{A}}=\left\{\mathbf{y} \in W^{1,1}\left(\Omega ; \mathbf{R}^{3}\right):\left.\mathbf{y}\right|_{\partial \Omega}=\mathbf{A} \mathbf{x}\right\}$, and a set $E$ is quasiconvex if it is the zero set $\varphi^{-1}(0)$ of a nonnegative quasiconvex function $\varphi$. We also have that

$$
K^{\mathrm{qc}}=\left\{\mathbf{A} \in M_{+}^{3 \times 3}: \inf _{\mathcal{A}_{\mathbf{A}}} I_{\theta}(\mathbf{y})=0\right\} .
$$


We can similarly define the polyconvexification $K^{p c}$ and the rank-one convexification $K^{r c}$ of a compact set $K \subset M_{+}^{3 \times 3}$ by

$$
\begin{aligned}
& K^{p c}=\left\{\mathbf{A} \in M^{3 \times 3}: \varphi(\mathbf{A}) \leq \max _{\mathbf{B} \in M^{3 \times 3}} \varphi(\mathbf{B}) \quad \text { for all polyconvex } \varphi\right\}, \\
& K^{r c}=\left\{\mathbf{A} \in M^{3 \times 3}: \varphi(\mathbf{A}) \leq \max _{\mathbf{B} \in M^{3 \times 3}} \varphi(\mathbf{B}) \quad \text { for all rank-one convex } \varphi\right\} .
\end{aligned}
$$

Clearly

$$
K^{r c} \subset K^{\mathrm{qc}} \subset K^{p c}
$$

Problem 16. Determine $K(\theta)^{\mathrm{qc}}$ for $\theta \leq \theta_{c}$.

For $\theta>\theta_{c}$ we have that $K(\theta)^{\mathrm{qc}}=K(\theta)$ (cf. Ball and James [1992]). For $\theta \leq \theta_{c}$ the problem is open. In particular, $K(\theta)^{\mathrm{qc}}$ is not known for the cubic-to-tetragonal case either when $\theta<\theta_{c}$ or $\theta=\theta_{c}$. Ball and James [1992] computed $K^{\mathrm{qc}}$ for the case of two wells

$$
K=\mathrm{SO}(3) \mathbf{U} \cup \mathrm{SO}(3) \mathbf{V},
$$

with $\mathbf{U}=\mathbf{U}^{T}>0, \mathbf{V}=\mathbf{V}^{T}>0, \operatorname{det} \mathbf{U}=\operatorname{det} \mathbf{V}$ and with $\operatorname{SO}(3) \mathbf{U}$ rankone connected to $\mathrm{SO}(3) \mathbf{V}$, which occurs for orthorhombic-to-monoclinic transformations. In this case by linear changes of variables we can assume that

$$
\mathbf{U}=\operatorname{diag}\left(\eta_{1}, \eta_{2}, \eta_{3}\right), \quad \mathbf{V}=\operatorname{diag}\left(\eta_{2}, \eta_{1}, \eta_{3}\right),
$$

where $\eta_{1}>0, \eta_{2}>0, \eta_{3}>0$ and $\eta_{1} \neq \eta_{2}$. (This includes the case $\mathbf{U}=$ $\mathbf{U}_{1}(\theta), \mathbf{V}=\mathbf{U}_{2}(\theta)$ of two tetragonal wells.) The answer in this case is (see Ball and James [2003]) that $K^{\mathrm{qc}}$ consists of those $\mathbf{A} \in M_{+}^{3 \times 3}$ such that

$$
\mathbf{A}^{T} \mathbf{A}=\left(\begin{array}{ccc}
a & c & 0 \\
c & b & 0 \\
0 & 0 & \eta_{3}^{2}
\end{array}\right),
$$

where $a b-c^{2}=\eta_{1}^{2} \eta_{2}^{2}, a+b+2|c| \leq \eta_{1}^{2}+\eta_{2}^{2}$. The proof is by calculating $K^{p c}$, showing that $K^{p c} \subset K^{r c}$, and using (4.3). Friesecke [2000] has announced that he can calculate $K(\theta)^{p c}, \theta<\theta_{c}$, in the cubic-to-tetragonal case. However, whether in general $K(\theta)^{p c}=K(\theta)^{\mathrm{qc}}$ is unknown. (Despite this, in their study of nonclassical austenite-martensite interfaces Ball and Carstensen [1999] were able to show that for $\theta<\theta_{c}$ the identity matrix is rank-one connected to $K(\theta)^{\mathrm{qc}}$ if and only if it is rank-one connected to $K(\theta)^{p c}$.)

Problem 17. For free-energy functions $\psi(\mathbf{A}, \theta)$ of elastic crystals, determine for which $\partial \Omega_{1} \subset \partial \Omega$ and $\mathbf{g}: \partial \Omega_{1} \rightarrow \mathbf{R}^{3}$ the minimum of

$$
I_{\theta}(\mathbf{y})=\int_{\Omega} \psi(D \mathbf{y}, \theta) d \mathbf{x}
$$

Version Dec 16, 2001

Edited by wgm : Typeset on 26 January $2002-9$ h 38 
in $\mathcal{A}=\left\{\mathbf{y} \in W^{1,1}\left(\Omega ; \mathbf{R}^{3}\right):\left.\mathbf{y}\right|_{\partial \Omega_{1}}=\mathbf{g}\right\}$ is attained, and for which it is not.

A solution to this problem (also to the corresponding problem including applied loads on $\partial \Omega_{2}$ ) would help clarify the validity of the hypothesis of Ball and James [1987] that the formation of microstructure is associated with non-attainment of minimum energy. For example, is non-attainment generic or exceptional?

It is probably overly optimistic to expect a general answer to Problem 17. A simpler special case for which the answer is in general unknown is when $\partial \Omega_{1}=\partial \Omega, \mathbf{g}(\mathbf{x})=\mathbf{A} \mathbf{x}$, and $\mathbf{A} \in K(\theta)^{\mathrm{qc}} \backslash K(\theta)$. In this case the problem is equivalent to asking whether for such $\mathbf{A}$ there exists a deformation $\mathbf{y}$ satisfying $\left.\mathbf{y}\right|_{\partial \Omega}=\mathbf{A x}$ and

$$
D \mathbf{y}(\mathbf{x}) \in K(\theta) \quad \text { a.e.. }
$$

For the corresponding two-well problem in which $K(\theta)$ is replaced by $K=$ $\mathrm{SO}(3) \mathbf{U} \cup \mathrm{SO}(3) \mathbf{V}$, with $\mathbf{U}, \mathbf{V}$ given by (4.4), there is no $\mathbf{y}$ with $\left.\mathbf{y}\right|_{\partial \Omega}=\mathbf{A x}$ and $D \mathbf{y}(\mathbf{x}) \in K$ a.e.. This non-attainment result was proved by Ball and Carstensen [1999] using the result of Ball and James [1991] that any $\mathbf{y}$ with $D \mathbf{y}(\mathbf{x}) \in K^{\mathrm{qc}}$ a.e. is a plane strain, the point being that a plane strain $\mathbf{y}$ cannot coincide with a linear mapping $\mathbf{A x}$ on the boundary of a three-dimensional region $\Omega$ unless $D \mathbf{y}(\mathbf{x})=\mathbf{A}$ a.e. . In the corresponding two-dimensional problem the answer is different. In fact, if $\Omega \subset \mathbf{R}^{2}$ and $K=\mathrm{SO}(2) \mathbf{U} \cup \mathrm{SO}(2) \mathbf{V}$, where

$$
\mathbf{U}=\operatorname{diag}\left(\eta_{1}, \eta_{2}\right), \quad \mathbf{V}=\operatorname{diag}\left(\eta_{2}, \eta_{1}\right),
$$

and $\eta_{1}>0, \eta_{2}>0$ then Müller and Šverák [1996] modified the theory of convex integration due to Gromov [1986] to show that there exists $\mathbf{y}$ with $\left.\mathbf{y}\right|_{\partial \Omega}=\mathbf{A x}, D \mathbf{y}(\mathbf{x}) \in K$ a.e. for any $\mathbf{A} \in K^{\mathrm{qc}}$. (For variations on the method see Dacorogna and Marcellini [1999], Müller and Sychev [2001], Sychev [2001] and Kirchheim [2001].) Whether these exotic minimizers exist in three dimensions, and if so whether they are physically relevant, is unclear. If, as seems likely, they do exist, then it is natural to ask whether they are admissible, in the sense that they can be obtained as limits of minimizers for a corresponding functional incorporating interfacial energy, for example

$$
I_{\theta}^{\varepsilon}(\mathbf{y})=\int_{\Omega}\left[\psi(D \mathbf{y}(\mathbf{x}), \theta)+\varepsilon^{2}\left|D^{2} \mathbf{y}(\mathbf{x})\right|^{2}\right] d \mathbf{x}
$$

in the limit $\varepsilon \rightarrow 0$.

For general information on the models and techniques described in this section see Ball and James [2003], Bhattacharya [2001], Hane [1997], Müller [1999], Luskin [1996] and Pedregal [1991, 2000]. 


\subsection{From three-dimensional elasticity to theories of rods and shells}

A rod is a three-dimensional body whose form is close to that of a curve in $\mathbf{R}^{3}$. We can describe a reasonably wide class of such bodies as those which occupy in a reference configuration the bounded domain

$$
\Omega_{h}=\left\{\mathbf{r}(s)+\mathbf{Q}(s)\left(0, \mathbf{x}^{\prime}\right): s \in(0, L), \mathbf{x}^{\prime} \in h D\right\},
$$

where $\mathbf{r}:(0, L) \rightarrow \mathbf{R}^{3}$ is a smooth embedded curve parametrized by arclength, so that $\left|\mathbf{r}^{\prime}(s)\right|=1$, where the cross-section $D \subset \mathbf{R}^{2}$ is a bounded domain with $0 \in D$, and where $\mathbf{Q}:(0, L) \rightarrow \mathrm{SO}(3)$ is a smooth mapping with $\mathbf{Q}(s) \mathbf{e}_{1}=\mathbf{r}^{\prime}(s)$ for each $s \in(0, L)$, which describes how the crosssection is rotated. The parameter $h>0$ measures the thickness of the rod. The simple case of an initially straight rod of circular cross-section corresponds to the choice $\mathbf{r}(s)=s \mathbf{e}_{1}, D=B(0,1) \subset \mathbf{R}^{2}, \mathbf{Q}(s)=\mathbf{1}$, so that $\Omega_{h}=(0, L) \mathbf{e}_{1} \times h D$.

A shell is a three-dimensional body whose form is close to that of a twodimensional surface. A class of such bodies consists of those occupying in a reference configuration the bounded domain

$$
\Omega_{h}=\left\{\mathbf{r}\left(s_{1}, s_{2}\right)+\tau \mathbf{n}\left(s_{1}, s_{2}\right):\left(s_{1}, s_{2}\right) \in S,|\tau| \leq h\right\},
$$

where $S \subset \mathbf{R}^{2}$ is a bounded domain, and $\mathbf{r}: S \rightarrow \mathbf{R}^{3}$ is a smooth embedded oriented surface with unit normal $\mathbf{n}\left(s_{1}, s_{2}\right)$. Here $h>0$ is the thickness of the shell. A plate is a flat shell, corresponding to

$$
\Omega_{h}=S \times(-h, h) .
$$

When $h$ is small, such thin rods and shells are traditionally described respectively by one-dimensional rod and two-dimensional shell models, in which the independent variables are respectively $(s, t)$ and $\left(s_{1}, s_{2}, t\right)$, where $t$ is time. There is an immense literature on the many such theories, well surveyed in the books of Antman [1995], and Ciarlet [1997, 2000]. However there are only the beginnings of a rigorous theory justifying such models with respect to three-dimensional elasticity.

Problem 18. Give a rigorous derivation of models of rods, plates and shells, showing that their solutions well approximate appropriate solutions to three-dimensional elasticity for small values of the thickness parameter $h$.

There seem to be no results of this type for dynamical theories of elasticity, so we concentrate on what is known for elastostatics. Here one would ideally like results showing that the solution sets for boundary-value value problems of three-dimensional elasticity converge as $h \rightarrow 0$ to corresponding sets for an appropriate rod or shell theory, together with appropriate error estimates. In passing to the limit $h \rightarrow 0$ other parameters such as 
loads may need to be scaled with $h$. Taking into account such scaling, one would like the convergence and error estimates to be uniform with respect to parameters such as loads entering the boundary conditions, so that in particular the description of buckling according to the three-dimensional theory could be correlated with that for the rod or shell theory identified. One of the many difficulties to be overcome in order to achieve such results is to understand how boundary-layers behave in the limit $h \rightarrow 0$. Such boundary layers will occur, for example, at the ends of a rod, where, according to Saint-Venant's principle one expects the limiting rod theory to see only resultant loads and moments applied to the ends. Higher-order corrections in $h$ can be expected to yield more sophisticated theories, for example involving numbers of directors (vectors depending on the independent variables giving a better description of the three-dimensional deformation).

An isolated theory that addresses some of these difficulties is Mielke's treatment Mielke [1988], Mielke [1990] of Saint-Venant's problem for an initially straight rod of uniform cross-section and prescribed resultant loads and moments at its two ends, in which via a six-dimensional centre manifold he identifies a Cosserat theory of rods whose solutions attract for long rods those of three-dimensional elasticity having uniformly small strains and the same resultant loads and moments. In this connection, for threedimensional nonlinear elasticity Ericksen [1977b,a, 1983] has derived equations describing beautiful semi-inverse solutions for helical deformations of a rod. For developments see Muncaster [1979, 1983], and for an existence theory for the corresponding problem defined on cross-sections see Ball [1977].

For plates with a St. Venant-Kirchhoff stored-energy function Monneau [2001] has devised a scheme which shows that for periodic boundary conditions and sufficiently small external forces, there is a solution of the threedimensional equilibrium equations which converges as $h \rightarrow 0$ to the solution of the corresponding Kirchhoff-Love plate theory, together with error estimates.

However, the principal method that has so far produced rigorous results of the desired type is $\Gamma$-convergence (see De Giorgi and Franzoni [1979], Dal Maso [1993]). The first application of $\Gamma$-convergence to nonlinear elasticity was that of Acerbi, Buttazzo and Percivale [1991], who used it to derive a one-dimensional model for an elastic string. Then Le Dret and Raoult [1995a,b, 1996, 1998] and Ben Belgacem [1997] used it to derive a corresponding two-dimensional membrane theory (see also Braides, Fonseca and Francfort [2000]). Le Dret and Raoult [2000] have also investigated which Cosserat theories of plates $\Gamma$-converge to the membrane theory limit as the thickness goes to zero. Bhattacharya and James [1999] used $\Gamma$-convergence to derive equations for thin films of martensitic material, an interesting conclusion being that in the two-dimensional theory there can be exact austenite-martensite interfaces (for developments see Shu [2000]).

In interesting recent work Friesecke, James, and Müller [2001] have de-

Version Dec 16, 2001 ....... Edited by wgm : Typeset on 26 January $2002-9$ h38 
rived a theory of nonlinear bending of plates starting from the nonlinear elastic energy

$$
I^{h}(\mathbf{y})=\int_{\Omega_{h}} W(D \mathbf{y}(\mathbf{x})) d \mathbf{x}
$$

where $\Omega_{h}$ is given by (4.5). This is a more delicate problem than for the membrane theory since for the boundary conditions for which the bending theory is expected to be valid, $I^{h}\left(\mathbf{y}_{h}\right)$ is expected to be of order $h^{3}$ for minimizers $\mathbf{y}_{h}$, whereas for boundary conditions leading to the membrane theory we expect that $I^{h}\left(\mathbf{y}_{h}\right)$ is of order $h$. Hence the limit $h \rightarrow 0$ corresponds to a singular perturbation. The corresponding bending theory has energy functional

$$
\frac{1}{24} \int_{S} Q_{2}(I I) d s_{1} d s_{2},
$$

where $I I$ denotes the second fundamental form

$$
I I_{i j}=\mathbf{n}_{, i} \cdot \mathbf{y}_{, j}, \quad \mathbf{n}=\mathbf{y}_{, 1} \wedge \mathbf{y}_{, 2},
$$

and where

$$
\begin{aligned}
& Q_{2}(\mathbf{A})=\min _{\mathbf{a} \in \mathbf{R}^{3}} Q_{3}\left(\mathbf{A}+\mathbf{a} \otimes \mathbf{e}_{3}+\mathbf{e}_{3} \otimes \mathbf{a}\right), \\
& Q_{3}(\mathbf{A})=D^{2} W(\mathbf{1})(\mathbf{A}, \mathbf{A}) .
\end{aligned}
$$

The proof is via a refinement of a rigidity result for $\mathrm{SO}(3)$ of John [1961, 1972a] (see also Kohn [1982]). John [1965, 1971] also rigorously obtains equations for shells of isotropic material, assuming that the radius of curvature of the shell is large and the maximum strain is uniformly small, providing interior estimates for the validity of the approximation. For other related work see Pantz [2000, 2001b]. For plates satisfying general boundary conditions one expects some theory incorporating both the membrane and bending cases, but the form this should take is unclear. For work in this direction see Ciarlet [2000] and Ciarlet and Roquefort [2000].

Acknowledgments: I am indebted to S. S. Antman, J. J. Bevan, K. Bhattacharya, C. M. Dafermos, G. Friesecke, R. D. James, M. Jungen, J. Kristensen, V. J. Mizel, O. Penrose, A. Raoult and A. Taheri for valuable suggestions and comments. The article was completed while I was visiting the Tata Institute for Fundamental Research in Bangalore, to whose members and staff I am grateful for their support and warm hospitality.

\section{References}

Acerbi E., G. Buttazzo and D. Percivale [1991], A variational definition of the strain energy for an elastic string. J. Elasticity, 25:137-148.

Version Dec 16, 2001 ....... Edited by wgm : Typeset on 26 January $2002-9$ h38 
Acerbi E., I. Fonseca and N. Fusco [1997], Regularity results for equilibria in a variational model of fracture. Proc. Royal Soc. Edinburgh, 127A:889-902.

Acerbi E., and N. Fusco [1984], Semicontinuity problems in the calculus of variations. Arch. Rational Mech. Anal., 86:125-145.

Acerbi E., and N. Fusco [1988], A regularity theorem for minimizers of quasiconvex integrals. Arch. Rational Mech. Anal., 99:261-281.

Ambrosio, L. [1989], Variational problems in SBV. Acta Appl. Math., 17:1-40.

Ambrosio, L. [1990], Existence theory for a new class of variational problems. Arch. Rational Mech. Anal., 111:291-322.

Ambrosio, L. and A. Braides [1995], Energies in SBV and variational models in fracture. In Homogenization and applications to material sciences (Nice 1995), volume 9 of GAKUTO Internat. Ser. Math. Sci. Appl., pages 1-22, Tokyo. Gakkötosho.

Ambrosio, L. N. Fusco and D. Pallara [1997], Partial regularity of free discontinuity sets II. Ann. Scuola Norm. Sup. Pisa Cl. Sci. (4), 24:39-62.

Ambrosio, L. N. Fusco and D. Pallara [2000], Functions of Bounded Variation and Free Discontinuity Problems. Oxford Mathematical Monographs. Oxford University Press.

Ambrosio, L. and D. Pallara [1997], Partial regularity of free discontinuity sets I. Ann. Scuola Norm. Sup. Pisa Cl. Sci. (4), 24:1-38.

Andrews, G. [1980], On the existence of solutions to the equation $u_{t t}=u_{x x t}+$ $\sigma\left(u_{x}\right)_{x}$. J. Differential Eqns, 35:200-231.

Antman, S. S. [1976], Ordinary differential equations of nonlinear elasticity. II. Existence and regularity theory for conservative boundary-value problem. Arch. Rational Mech. Anal., 61:353-393.

Antman, S. S. [1983], The influence of elasticity on analysis: Modern developments. Bull. Amer. Math. Soc., 9:267-291.

Antman, S. S. [1995], Nonlinear Problems of Elasticity, volume 107 of Applied Mathematical Sciences. Springer-Verlag, New York.

Antman, S. S. and P. V. Negrón-Marrero [1987], The remarkable nature of radially symmetric equilibrium states of aeolotropic nonlinearly elastic bodies. $J$. Elasticity, 18:131-164.

Antman, S. S. and J. E. Osborn [1979], The principle of virtual work and integral laws of motion. Arch. Rational Mech. Anal., 69:231-262.

Antman, S. S. and T. Seidman [1996], Quasilinear hyperbolic-parabolic equations of one-dimensional viscoelasticity. J. Differential Eqns, 124:132-185.

Ball, J. M. [1977], Constitutive inequalities and existence theorems in nonlinear elastostatics. In R.J. Knops, editor, Nonlinear Analysis and Mechanics, HeriotWatt Symposium, Vol. 1. Pitman.

Ball, J. M. [1977a], Convexity conditions and existence theorems in nonlinear elasticity. Arch. Rational Mech. Anal., 63:337-403.

Ball, J. M. [1980], Strict convexity, strong ellipticity, and regularity in the calculus of variations. Proc. Camb. Phil. Soc., 87:501-513.

Version Dec 16, 2001 ....... Edited by wgm : Typeset on 26 January $2002-9$ h38 
Ball, J. M. [1981], Global invertibility of Sobolev functions and the interpenetration of matter. Proc. Royal Soc. Edinburgh, 88A:315-328.

Ball, J. M. [1981a], Remarques sur l'existence et la régularité des solutions d'élastostatique non linéaire. In H. Berestycki and H. Brezis, editors, Recent Contributions to Nonlinear Partial Differential Equations. Pitman.

Ball, J. M. [1982], Discontinuous equilibrium solutions and cavitation in nonlinear elasticity. Phil. Trans. Royal Soc. London A, 306:557-611.

Ball, J. M. [1984], Differentiability properties of symmetric and isotropic functions. Duke Math. J., 51:699-728.

Ball, J. M. [1984a], Minimizers and the Euler-Lagrange equations. In Trends and applications of pure mathematics to mechanics (Palaiseau, 1983), pages 1-4. Springer, Berlin.

Ball, J. M. [1986], Minimizing sequences in thermomechanics. In Proc. Meeting on "Finite Thermoelasticity", pages 45-54, Roma. Accademia Nazionale dei Lincei.

Ball, J. M. [1989], A version of the fundamental theorem for Young measures. In M. Rascle, D. Serre and M. Slemrod, editors, Proceedings of conference on "Partial differential equations and continuum models of phase transitions," pages 3-16. Springer Lecture Notes in Physics. No. 359.

Ball, J. M. [1992], Dynamic energy minimization and phase transformations in solids. In Proceedings of ICIAM 91. SIAM.

Ball, J. M. [1996], Nonlinear elasticity and materials science; a survey of some recent developments. In P.J. Aston, editor, Nonlinear Mathematics and Its Applications, pages 93-119. Cambridge University Press.

Ball, J. M. [1996a], Review of Nonlinear Problems of Elasticity, by Stuart S. Antman. Bull. Amer. Math. Soc., 33:269-276.

Ball, J. M. [1998], The calculus of variations and materials science. Quart. Appl. Math., 56:719-740.

Ball, J. M. [2001], Singularities and computation of minimizers for variational problems. In R. DeVore, A. Iserles and E. Suli, editors, Foundations of Computational Mathematics. Cambridge University Press.

Ball, J. M. and C. Carstensen [1999], Compatibility conditions for microstructures and the austenite-martensite transition. Materials Science $\mathscr{G}$ Engineering A, 273-275:231-236.

Ball, J. M. , C. Chu and R. D. James [1995], Hysteresis during stress-induced variant rearrangement. $J$. de Physique $I V$, C8:245-251.

Ball, J. M. , C. Chu and R. D. James [2002], Metastability and martensite. In preparation.

Ball, J. M. , P. J. Holmes, R. D. James, R. L. Pego and P. J. Swart [1991], On the dynamics of fine structure. J. Nonlinear Sci., 1:17-90.

Ball, J. M. and R. D. James [2003], From Microscales to Macroscales in Materials. Book, in preparation.

Ball, J. M. and R. D. James [2002], Incompatible sets of gradients and metastability. In preparation.

Version Dec 16, $2001 \ldots \ldots$. Edited by wgm : Typeset on 26 January $2002-9$ h38 
Ball, J. M. and R. D. James [1987], Fine phase mixtures as minimizers of energy. Arch. Rational Mech. Anal., 100:13-52.

Ball, J. M. and R. D. James [1991], A characterization of plane strain. Proc. Roy. Soc. London A, 432:93-99.

Ball, J. M. and R. D. James [1992], Proposed experimental tests of a theory of fine microstructure, and the two-well problem. Phil. Trans. Roy. Soc. London $A, 338: 389-450$.

Ball, J. M. and J. E. Marsden [1984], Quasiconvexity at the boundary, positivity of the second variation, and elastic stability. Arch. Rational Mech. Anal., 86:251-277.

Ball, J. M. and V. J. Mizel [1985], One-dimensional variational problems whose minimizers do not satisfy the Euler-Lagrange equations. Arch. Rational Mech. Anal., 90:325-388.

Ball, J. M. and F. Murat [1984], $W^{1, p}$-quasiconvexity and variational problems for multiple integrals. J. Functional Analysis, 58:225-253.

Bauman, P., N. C. Owen and D. Phillips [1991], Maximal smoothness of solutions to certain Euler-Lagrange equations from nonlinear elasticity. Proc. Royal Soc. Edinburgh, 119A:241-263.

Bauman, P., N. C. Owen and D. Phillips [1991a], Maximum principles and a priori estimates for a class of problems from nonlinear elasticity. Annales de l'Institut Henri Poincaré - Analyse non linéaire, 8:119-157.

Bauman, P., N. C. Owen and D. Phillips [1992], Maximum principles and a priori estimates for an incompressible material in nonlinear elasticity. Comm. in Partial Diff. Eqns, 17:1185-1212.

Bauman, P. and D. Phillips [1994], Univalent minimizers of polyconvex functionals in 2 dimensions. Arch. Rational Mech. Anal., 126:161-181.

Ben Belgacem, H. [1997], Une méthode de $\Gamma$-convergence pour un modèle de membrane non linéaire. C. R. Acad. Sci. Paris Sér. I Math., 324:845-849.

Bhattacharya, K. [2001], Microstructure of martensite. A continuum theory with applications to the shape-memory effect. Oxford University Press (to appear).

Bhattacharya, K. and R. D. James [1999], A theory of thin films of martensitic materials with applications to microactuators. J. Mech. Phys. Solids, 47:531576 .

Bianchini, S. and A. Bressan [2001], A center manifold technique for tracing viscous waves. Preprint.

Blanc, X., C. Le Bris and P.-L. Lions [2001], Convergence de modèles moléculaires vers des modèles de mécanique des milieux continus. C. R. Acad. Sci. Paris Sér. I Math., 332:949-956.

Bourdin, B., G. A. Francfort and J.-J. Marigo [2000], Numerical experiments in revisited brittle fracture. J. Mech. Phys. Solids, 48:797-826.

Braides, A. [1994], Loss of polyconvexity by homogenization. Arch. Rational Mech. Anal., 127:183-190.

Braides A. [1998], Approximation of Free-Discontinuity Problems, volume 1694 of Lecture Notes in Mathematics. Springer-Verlag, Berlin.

Version Dec 16, 2001 ....... Edited by wgm : Typeset on 26 January $2002-9$ h38 
Braides A. and A. Coscia [1993], A singular perturbation approach to variational problems in fracture mechanics. Math. Models Methods Appl. Sci., 3:303-340.

Braides A. and A. Coscia [1994], The interaction between bulk energy and surface energy in multiple integrals. Proc. Royal Soc. Edinburgh, 124A:737-756.

Braides A., I. Fonseca and G. Francfort [2000], 3D-2D asymptotic analysis for inhomogeneous thin films. Indiana Univ. Math. J., 49:1367-1404.

Braides A. and M. S. Gelli [2001a], Limits of discrete systems with long-range interactions. Preprint.

Braides A. and M. S. Gelli [2001b], Limits of discrete sytems without convexity hypotheses. Preprint.

Braides A., G. Dal Maso and A. Garroni [1999], Variational formulation for softening phenomena in fracture mechanics: the one-dimensional case. Arch. Rational Mech. Anal., 146:23-58.

Bressan, A. [1988], Contractive metrics for nonlinear hyperbolic systems. Indiana J. Math., 37:409-421.

Bressan, A. [1995], The unique limit of the Glimm scheme. Arch. Rational Mech. Anal., 130:205-230.

Bressan, A. [2000], Hyperbolic Systems of Conservation Laws. Oxford Lecture Series in Mathematics and Its Applications. Oxford University Press.

Bressan, A. and R. M. Colombo [1995], The semigroup generated by $2 \times 2$ conservation laws. Arch. Rational Mech. Anal., 133:1-75.

Bressan, A., G. Crasta and B. Piccoli [2000], Well-posedness of the Cauchy problem for $n \times n$ systems of conservation laws. Mem. Amer. Math. Soc, 146(694).

Bressan, A. and P. G. Le Floch [1997], Uniqueness of weak solutions to hyperbolic systems of conservation laws. Arch. Rational Mech. Anal., 140:301-317.

Bressan, A. and P. Goatin [1999], Oleinik type estimates and uniqueness for $n \times n$ conservation laws. J. Differential Eqns, 156:26-49.

Bressan, A. and M. Lewicka [2000], A uniqueness condition for hyperbolic systems of conservation laws. Discrete Contin. Dynam. Systems, 6:673-682.

Bressan, A., T.-P. Liu and T. Yang [1999], $L_{1}$ stability estimates for $n \times n$ conservation laws. Arch. Rational Mech. Anal., 149:1-22.

Buttazzo, G. [1995], Energies on BV and variational models in fracture mechanics. In Curvature flows and related topics (Levico, 1994), volume 5 of GAKUTO Internat. Ser. Math. Sci. Appl., pages 25-36, Tokyo. Gakkötosho.

Buttazzo, G. and M. Belloni [1995], A survey on old and recent results about the gap phenomenon. In Recent Developments in Well-Posed Variational Problems, pages 1-27, edited by R. Lucchetti and J. Revalski, Kluwer Academic Publishers, Dordrecht.

Catto, I., C. Le Bris and P.-L. Lions [1998], The Mathematical Theory of Thermodynamic Limits: Thomas-Fermi Type Models. Oxford University Press.

Cherepanov, G. P., editor [1998], Fracture. Krieger, Malabar, Fl.

Version Dec 16, 2001 ....... Edited by wgm : Typeset on 26 January $2002-9$ h38 
Chillingworth, D. R. J., J. E. Marsden and Y. H. Wan [1982], Symmetry and bifurcation in three-dimensional elasticity, I. Arch. Rational Mech. Anal., 80:295331.

Chillingworth, D. R. J., J. E. Marsden and Y. H. Wan [1983], Symmetry and bifurcation in three-dimensional elasticity, II. Arch. Rational Mech. Anal., 83:363-395.

Chlebík, M. and B. Kirchheim [2001], Rigidity for the four gradient problem (to appear).

Chu, C. and R. D. James [1993], Biaxial loading experiments on Cu-Al-Ni single crystals. In Experiments in Smart Materials and Structures, pages 61-69. ASME. AMD-Vol. 181.

$\mathrm{Chu}$, C. and R. D. James [1995], Analysis of microstructures in Cu-14.0\%Al$3.9 \% \mathrm{Ni}$ by energy minimization. J. de Physique IV, C8:143-149.

Ciarlet, P. G. [2000], Un modèle bi-dimensionnel non linéaire de coque analogue à celui de W. T. Koiter. C. R. Acad. Sci. Paris Sér. I Math., 331:405-410.

Ciarlet, P. G. [1988], Mathematical Elasticity, Vol.I: Three-Dimensional Elasticity. North-Holland.

Ciarlet, P. G. [1997], Mathematical Elasticity. Vol. II: Theory of Plates. NorthHolland Publishing Co., Amsterdam.

Ciarlet, P. G. [2000], Mathematical Elasticity. Vol. III: Theory of Shells. NorthHolland Publishing Co., Amsterdam.

Ciarlet, P. G. and J. Nečas [1985], Unilateral problems in nonlinear threedimensional elasticity. Arch. Rational Mech. Anal., 87:319-338.

Ciarlet, P. G. and A. Roquefort [2000], Justification d'un modèle bi-dimensionnel non linéaire de coque analogue à celui de W. T. Koiter. C. R. Acad. Sci. Paris Sér. I Math., 331(5):411-416.

Coleman, B. D. and E.H. Dill [1973], On thermodynamics and the stability of motion of materials with memory. Arch. Rational Mech. Anal., 51:1-53.

Coleman, B. D. and W. Noll [1963], The thermodynamics of elastic materials with heat conduction and viscosity. Arch. Rational Mech. Anal., 13:167-178.

Dacorogna, B. [1982], Quasiconvexity and relaxation of non convex variational problems. J. Funct. Anal., 46:102-118.

Dacorogna, B. and P. Marcellini [1999], Implicit Partial Differential Equations. Birkhäuser Boston Inc., Boston, MA.

Dafermos, C. M. [1969], The mixed initial boundary-value problem for the equations of nonlinear one-dimensional viscoelasticity. J. Differential Eqns, 6:71-86.

Dafermos, C. M. [1972], Polygonal approximations of solutions of the initial value problem for a conservation law. J. Math. Anal. Appl., 38:33-41.

Dafermos, C. M. [1996], Entropy and the stability of classical solutions of hyperbolic systems of conservation laws. In Recent Mathematical Methods in Nonlinear Wave Propagation (Montecatini Terme, 1994), volume 1640 of Lecture Notes in Math., pages 48-69, Berlin. Springer.

Dafermos, C. M. [2000], Hyperbolic Conservation Laws in Continuum Physics, volume 325 of Grundlehren der Mathematischen Wissenschaften. Springer.

Version Dec 16, 2001 ....... Edited by wgm : Typeset on 26 January $2002-9$ h38 
Dafermos, C. M. and W. J. Hrusa [1985], Energy methods for quasilinear hyperbolic initial boundary-value problems. Arch. Rational Mech. Anal., 87:267-292.

Dal Maso, G. [1993], An Introduction to $\Gamma$-convergence. Birkhäuser Boston Inc., Boston, MA.

De Giorgi, E. and T. Franzoni [1979], On a type of variational convergence. In Proceedings of the Brescia Mathematical Seminar, Vol. 3 (Italian), pages 63-101, Milan. Univ. Cattolica Sacro Cuore.

Deam, R. T. and S. F. Edwards [1976], The theory of rubber elasticity. Philos. Trans. Roy. Soc. London Ser. A, 280:317-353.

Demoulini, S. [2000], Weak solutions for a class of nonlinear systems of viscoelasticity. Arch. Rational Mech. Anal., 155:299-334.

Demoulini, S., D. M. A. Stuart and A.E. Tzavaras [2000], Construction of entropy solutions for one-dimensional elastodynamics via time discretisation. Ann. Inst. H. Poincaré Anal. Non Linéaire, 17:711-731.

Demoulini, S., D. M. A. Stuart and A.E. Tzavaras [2001], A variational approximation scheme for three-dimensional elastodynamics with polyconvex energy. Arch. Rational Mech. Anal., 157:325-344.

DiPerna, R. J. [1983], Convergence of approximate solutions of conservation laws. Arch. Rational Mech. Anal., 82:27-70.

DiPerna, R. J. [1985], Compensated compactness and general systems of conservation laws. Trans. A.M.S., 292:283-420.

Duhem, P. [1911], Traité d'Énergetique ou de Thermodynamique Générale. Gauthier-Villars, Paris.

Ebin, D. G. [1993], Global solutions of the equations of elastodynamics of incompressible neo-Hookean materials. Proc. Nat. Acad. Sci. U.S.A., 90:3802-3805.

Ebin, D. G. [1996], Global solutions of the equations of elastodynamics for incompressible materials. Electron. Res. Announc. Amer. Math. Soc., 2:50-59 (electronic).

Ebin, D. G. and R.A. Saxton [1986], The initial value problem for elastodynamics of incompressible bodies. Arch. Rational Mech. Anal., 94:15-38.

Ebin, D. G. and S.R. Simanca [1990], Small deformations of incompressible bodies with free boundary. Comm. Partial Differential Equations, 15:1589-1616.

Ebin, D. G. and S.R. Simanca [1992], Deformations of incompressible bodies with free boundaries. Arch. Rational Mech. Anal., 120:61-97.

Edwards, S. F. and T.A. Vilgis [1988], The tube model theory of rubber elasticity. Rep. Progr. Phys., 51:243-297.

Ericksen, J. L. [1966], Thermoelastic stability. In Proc $5^{\text {th }}$ National Cong. Appl. Mech., pages 187-193.

Ericksen, J. L. [1977b], On the formulation of St.-Venant's problem. In Nonlinear analysis and mechanics: Heriot-Watt Symposium (Edinburgh, 1976), Vol. I, pages 158-186. Res. Notes in Math., No. 17. Pitman, London.

Ericksen, J. L. [1977b], Special topics in elastostatics. In C.-S. Yih, editor, Advances in Applied Mechanics, volume 17, pages 189-244. Academic Press.

Version Dec 16, 2001 ....... Edited by wgm : Typeset on 26 January $2002-9$ h38 
Ericksen, J. L. [1983], Ill-posed problems in thermoelasticity theory. In Proceedings of a NATO/London Mathematical Society advanced study institute held in Oxford, July 25-August 7, 1982, pages 71-93. D. Reidel Publishing Co., Dordrecht.

Euler, L. [1744], Additamentum I de curvis elasticis, methodus inveniendi lineas curvas maximi minimivi proprietate gaudentes. Bousquent, Lausanne. In Opera Omnia I, Vol. 24, 231-297.

Evans, L. C. [1986], Quasiconvexity and partial regularity in the calculus of variations. Arch. Rational Mech. Anal., 95:227-268.

Evans, L. C. and R. F. Gariepy [1987], Some remarks concerning quasiconvexity and strong convergence. Proc. Roy. Soc. Edinburgh, 106A:53-61.

Fefferman, C. [1985], The thermodynamic limit for a crystal. Comm. Math. Phys., 98(3):289-311.

Foccardi, M. and M. S. Gelli [2001], A finite-differences approximation of fracture energies for non-linear elastic materials. Preprint.

Fonseca, I. [1988], The lower quasiconvex envelope of the stored energy function of an elastic crystal. J. Math. Pures Appl., 67:175-195.

Fonseca, I. and W. Gangbo [1995], Local invertibility of Sobolev functions. SIAM J. Math. Anal., 26:280-304.

Foss, M. [2001], On Lavrentiev's Phenomenon. PhD thesis, Carnegie-Mellon University.

Francfort, G. A. and J.-J. Marigo [1998], Revisiting brittle fracture as an energy minimization problem. J. Mech. Phys. Solids, 46:1319-1342.

Friesecke, G. [2000], personal communication.

Friesecke, G. and G. Dolzmann [1997], Implicit time discretization and global existence for a quasi-linear evolution equation with nonconvex energy. SIAM J. Math. Anal., 28:363-380.

Friesecke, G. and R. D. James [2000], A scheme for the passage from atomic to continuum theory for thin films, nanotubes and nanorods. J. Mech. Phys. Solids, 48:1519-1540.

Friesecke, G., R. D. James and S. Müller [2001], Rigorous derivation of nonlinear plate theory and geometric rigidity. C. R. Acad. Sci. Paris Sér. I Math. (to appear).

Friesecke, G. and J. B. McLeod [1996], Dynamics as a mechanism preventing the formation of finer and finer microstructure. Arch. Rational Mech. Anal., 133:199-247.

Friesecke, G. and J. B. McLeod [1997], Dynamic stability of non-minimizing phase mixtures. Proc. Roy. Soc. London Ser. A, 453:2427-2436.

Friesecke, G. and F. Theil [2001], Validity and failure of the Cauchy-Born hypothesis in a 2D mass-spring lattice. Preprint.

Giaquinta, M., G. Modica and J. Souček [1989], Cartesian currents, weak diffeomorphisms and existence theorems in nonlinear elasticity. Arch. Rational Mech. Anal., 106:97-159. Addendum, ibid., 109:385-392, 1990.

Version Dec 16, 2001 Edited by wgm : Typeset on 26 January $2002-9 \mathrm{~h} 38$ 
Giaquinta, M., G. Modica and J. Souček [1994], A weak approach to finite elasticity. Calc. Var. Partial Differential Equations, 2:65-100.

Giaquinta, M., G. Modica and J. Souček [1998], Cartesian Currents in the Calculus of Variations. Volumes I, II. Springer-Verlag, Berlin. Cartesian currents.

Glimm, J. [1965], Solutions in the large for nonlinear hyperbolic systems of equations. Comm. Pure Appl. Math., 18:697-715.

Green, A. E. and J. E. Adkins [1970], Large Elastic Deformations. Oxford Univ. Press, 2nd edition.

Green, A. E. and W. Zerna [1968], Theoretical Elasticity. Clarendon Press, Oxford, 2nd edition.

Greenberg, J. M. , R. C. MacCamy and V. J. Mizel [1967], On the existence, uniqueness, and stability of solutions of the equation $\sigma^{\prime}\left(u_{x}\right) u_{x x}+\lambda u_{x t x}=$ $\rho_{0} u_{t t}$. J. Math. Mech., 17:707-728, 1967/1968.

Gromov, M. [1986], Partial Differential Relations. Springer-Verlag, Berlin.

Gurtin, M. E. [1981], Topics in Finite Elasticity. SIAM, 1981.

Hane, K. [1997], Microstructures in Thermoelastic Martensites. PhD thesis, Department of Aerospace Engineering and Mechanics, University of Minnesota.

Hao, W., S. Leonardi and J. Nečas [1996], An example of irregular solution to a nonlinear Euler-Lagrange elliptic system with real analytic coefficients. Ann. Scuola Norm. Sup. Pisa Cl. Sci. (4), 23:57-67.

Healey, T. J. [2000], Global continuation in displacement problems of nonlinear elastostatics via the Leray-Schauder degree. Arch. Rational Mech. Anal., 152:273-28.

Healey, T. J. and P. Rosakis [1997], Unbounded branches of classical injective solutions to the forced displacement problem in nonlinear elastostatics. $J$. Elasticity, 49:65-78.

Healey, T. J. and H. Simpson [1998], Global continuation in nonlinear elasticity. Arch. Rational Mech. Anal., 143:1-28.

Hrusa, W. J. and M. Renardy [1988], An existence theorem for the Dirichlet problem in the elastodynamics of incompressible materials. Arch. Rational Mech. Anal., 102:95-117. Corrections ibid 110:373-375,1990.

Hughes, T. J. R., T. Kato and J. E. Marsden [1977], Well-posed quasilinear hyperbolic systems with applications to nonlinear elastodynamics and general relativity. Arch. Rational Mech. Anal., 63:273-294.

James, R. D. and S. J. Spector [1991], The formation of filamentary voids in solids. J. Mech. Phys. Solids, 39:783-813.

Jiang, S. and R. Racke [2000], Evolution equations in thermoelasticity. Chapman \& Hall/CRC, Boca Raton, FL.

John, F. [1961], Rotation and strain. Comm. Pure Appl. Math., 14:391-413.

John, F. [1965], Estimates for the derivatives of the stresses in a thin shell and interior shell equations. Comm. Pure Appl. Math., 18:235-267.

John, F. [1971], Refined interior equations for thin elastic shells. Comm. Pure Appl. Math., 24:583-615.

Version Dec 16, 2001 ....... Edited by wgm : Typeset on 26 January $2002-9$ h38 
John, F. [1972a], Bounds for deformations in terms of average strains. In Inequalities, III (Proc. Third Sympos., Univ. California, Los Angeles, Calif., 1969; dedicated to the memory of Theodore S. Motzkin), pages 129-144. Academic Press, New York.

John, F. [1972b], Uniqueness of non-linear elastic equilibrium for prescribed boundary displacements and sufficiently small strains. Comm. Pure Appl. Math., 25:617-634.

John, F. [1988], Almost global existence of elastic waves of finite amplitude arising from small initial disturbances. Comm. Pure Appl. Math., 41:615-666.

Kato, T. [1985], Abstract Differential Equations and Nonlinear Mixed Problems. Lezioni Fermi. Scuola Normale Superiore, Pisa; Accademia Nazionale dei Lincei, Rome.

Kinderlehrer, D. and P. Pedregal [1991], Characterizations of Young measures generated by gradients. Arch. Rational Mech. Anal., 115:329-365.

Kinderlehrer, D. and P. Pedregal [1994], Gradient Young measures generated by sequences in Sobolev spaces. J. Geom. Anal., 4:59-90.

Kirchheim, B. [2001], Deformations with finitely many gradients and stability of quasiconvex hulls. C. R. Acad. Sci. Paris Sér. I Math., 332:289-294.

Knops, R. J. and C.A. Stuart [1984], Quasiconvexity and uniqueness of equilibrium solutions in nonlinear elasticity. Arch. Rational Mech. Anal., 86:233-249.

Knops, R. J. and E. W. Wilkes [1973], Theory of elastic stability. In S. Flugge, editor, Encyclopedia of Physics, volume VIa/1-4. Springer-Verlag, Berlin.

Kohn, R. V. [1982], New integral estimates for deformations in terms of their nonlinear strains. Arch. Rational Mech. Anal., 78:131-172.

Koiter, W. T. [1976], A basic open problem in the theory of elastic stability. In Applications of Methods of Functional Analysis to Problems in Mechanics (Joint Sympos., IUTAM/IMU, Marseille, 1975), pages 366-373. Lecture Notes in Math., 503. Springer, Berlin.

Kristensen, J. [1994], Lower Semicontinuity of Variational Integrals. PhD thesis, Technical University of Lyngby.

Kristensen, J. [1999], On the non-locality of quasiconvexity. Ann. Inst. H. Poincaré, Anal. Non Linéaire, 16:1-13.

Kristensen, J. and A. Taheri [2001], Partial regularity of strong local minimisers. Preprint.

Lazzeri, A. and C. B. Bucknall [1995], Applications of a dilatational yielding model to rubber-toughened polymers. Polymer, 36:2895-2902.

Le Dret, H. [1990], Sur les fonctions de matrices convexes et isotropes. C. $R$. Acad. Sci. Paris Sér. I Math., 310:617-620.

Le Dret, H. and A. Raoult [1995a], From three-dimensional elasticity to nonlinear membranes. In Asymptotic methods for elastic structures (Lisbon, 1993), pages 89-102. de Gruyter, Berlin.

Le Dret, H. and A. Raoult [1995b], The nonlinear membrane model as variational limit of nonlinear three-dimensional elasticity. J. Math. Pures Appl., 74:549578 .

Version Dec 16, $2001 \ldots \ldots$. Edited by wgm : Typeset on 26 January $2002-9 \mathrm{~h} 38$ 
Le Dret, H. and A. Raoult [1996], The membrane shell model in nonlinear elasticity: a variational asymptotic derivation. J. Nonlinear Sci., 6:59-84.

Le Dret, H. and A. Raoult [1998], From three-dimensional elasticity to the nonlinear membrane model. In Nonlinear partial differential equations and their applications. Collège de France Seminar, Vol. XIII (Paris, 1994/1996), pages 192-206. Longman, Harlow.

Le Dret, H. and A. Raoult [2000], Variational convergence for nonlinear shell models with directors and related semicontinuity and relaxation results. Arch. Ration. Mech. Anal., 154:101-134.

Lieb, E. H. and B. Simon [1977], The Thomas-Fermi theory of atoms, molecules and solids. Adv. Math., 23:22-116.

Lin, P. [1990], Maximization of the entropy for an elastic body free of surface traction. Arch. Rational Mech. Anal., 112:161-191.

Liu, T.-P. [1977], initial boundary-value problems in gas dynamics. Arch. Rational Mech. Anal., 64:137-168.

Liu, T.-P. [1981], Admissible solutions of hyperbolic conservation laws. Memoirs $A M S, 30(240)$.

Liu, T.-P. and T. Yang [1999a], $L_{1}$ stability for $2 \times 2$ systems of hyperbolic conservation laws. J. Amer. Math. Soc., 12:729-774.

Liu, T.-P. and T. Yang [1999b], $L_{1}$ stability of conservation laws with coinciding hugoniot and characteristic curves. Indiana Univ. Math. J, 48:237-247.

Liu, T.-P. and T. Yang [1999c], Well-posedness theory for hyperbolic conservation laws. Comm. Pure Appl. Math, 52:1553-1586.

Love, A. E. H. [1927], A treatise on the mathematical theory of elasticity. Cambridge University Press, fourth revised enlarged edition, Reprinted by Dover, New York, 1944.

Luskin, M. [1996], On the computation of crystalline microstructure. Acta $\mathrm{Nu}$ merica, 5:191-258.

Marsden, J. E. and T.J.R. Hughes [1983], Mathematical Foundations of Elasticity. Prentice-Hall.

Meisters, G. H. and C. Olech [1963], Locally one-to-one mappings and a classical theorem on Schlicht functions. Duke Math. J., 30:63-80.

Mielke, A. [1988], Saint-Venant's problem and semi-inverse solutions in nonlinear elasticity. Arch. Rational Mech. Anal., 102:205-229. Corrigendum ibid. 110::351-352, 1990.

Mielke, A. [1990], Normal hyperbolicity of center manifolds and Saint-Venant's principle. Arch. Rational Mech. Anal., 110:353-372.

Mizel, V. J., M. Foss and W. J. Hrusa [2002], The Lavrentiev gap phenomenon in nonlinear elasticity. (to appear).

Monneau, R. [2001], Justification de la théorie non linéaire de Kirchhoff-Love, comme application d'une nouvelle méthode d'inversion singulière. C. R. Acad. Sci. Paris Sér. I Math. (to appear).

Morrey, C. B. [1952], Quasi-convexity and the lower semicontinuity of multiple integrals. Pacific J. Math., 2:25-53.

Version Dec 16, 2001 ....... Edited by wgm : Typeset on 26 January $2002-9$ h38 
Müller, S. [1988], Weak continuity of determinants and nonlinear elasticity. $C$. R. Acad. Sci. Paris Sér. I Math., 307:501-506.

Müller, S. [1999], Variational methods for microstructure and phase transitions. In Calculus of variations and geometric evolution problems, volume 1713 of Lecture Notes in Math., pages 85-210. Springer, Berlin.

Müller, S., T. Qi and B.S. Yan [1994], On a new class of elastic deformations not allowing for cavitation. Ann. Inst. Henri Poincaré, Analyse Nonlinéaire, $11: 217-243$.

Müller, S. and S. J. Spector [1995], An existence theory for nonlinear elasticity that allows for cavitation. Arch. Rational Mech. Anal., 131:1-66.

Müller, S. and V. Šverák [1996], Attainment results for the two-well problem by convex integration. In J. Jost, editor, Geometric analysis and the calculus of variations, pages 239-251. International Press.

Müller, S. and V. Šverák [2001], Convex integration for Lipschitz mappings and counterexamples to regularity. Annals of Math. (to appear).

Müller, S. and M. A. Sychev [2001], Optimal existence theorems for nonhomogeneous differential inclusions. J. Funct. Anal., 181:447-475.

Muncaster, R. G. [1979], Saint-Venant's problem in nonlinear elasticity: a study of cross sections. In Nonlinear analysis and mechanics: Heriot-Watt Symposium, Vol. IV, pages 17-75. Pitman, Boston, Mass.

Muncaster, R. G. [1983], Saint-Venant's problem for slender prisms. Utilitas Math., 23:75-101, 1983.

Nečas, J. [1977], Example of an irregular solution to a nonlinear elliptic system with analytic coefficients and conditions for regularity. In Theory of Nonlinear Operators, pages 197-206, Berlin. Akademie-Verlag.

Ogden, R. W. [1972a], Large deformation isotropic elasticity - on the correlation of theory and experiment for incompressible rubberlike solids. Proc. Roy. Soc. London A, 326:562-584.

Ogden, R. W. [1972b], Large deformation isotropic elasticity: on the correlation of theory and experiment for compressible rubberlike solids. Proc. Roy. Soc. London A, 328:567-583.

Ogden, R. W. [1984], Nonlinear Elastic Deformations. Ellis Horwood.

Pantz, O. [2000], Dérivation des modèles de plaques membranaires non linéaires à partir de l'élasticité tri-dimensionnelle. C. R. Acad. Sci. Paris Sér. I Math., 331:171-174.

Pantz, O. [2001a], Quelques Problèmes de Modélisation en Élasticité Nonlinéaire. $\mathrm{PhD}$ thesis, Université Paris 6.

Pantz, O. [2001b], Une justification partielle du modèle de plaque en flexion par Г-convergence. C. R. Acad. Sci. Paris Sér. I Math., 332:587-592.

Pedregal, P. [1991], Parametrized Measures and Variational Principles, volume 30 of Progress in nonlinear differential equations and their applications. Birkhäuser, Basel.

Pedregal, P. [1994], Jensen's inequality in the calculus of variations. Differential Integral Equations, 7:57-72.

Version Dec 16, 2001 ....... Edited by wgm : Typeset on 26 January $2002-9$ h38 
Pedregal, P. [2000], Variational Methods in Nonlinear Elasticity. SIAM, Philadelphia.

Pego, R. L. [1987], Phase transitions in one-dimensional nonlinear viscoelasticity: admissibility and stability. Arch. Rational Mech. Anal., 97:353-394.

Penrose, O. [2001], Statistical mechanics of nonlinear elasticity. Markov Processes and Related Fields. (to appear).

Pericak-Spector, K. A. and S. J. Spector [1997], Dynamic cavitation with shocks in nonlinear elasticity. Proc. Roy. Soc. Edinburgh, 127A:837-857.

Phillips, D. [2001], On one-homogeneous solutions to elliptic systems in two dimensions. C. R. Acad. Sci. Paris Sér. I Math. (to appear).

Phillips, R. [2001]. Crystals, defects and microstructures. Cambridge University Press.

Polignone, D. A. and C. O. Horgan [1993a], Cavitation for incompressible anisotropic non-linearly elastic spheres. J. Elasticity, 33:27-65.

Polignone, D. A. and C. O. Horgan [1993b], Effects of material anisotropy and inhomogeneity on cavitation for composite incompressible anisotropic nonlinearly elastic spheres. Internat. J. Solids Structures, 30:3381-3416.

Post, K. D. E. and J. Sivaloganathan [1997], On homotopy conditions and the existence of multiple equilibria in finite elasticity. Proc. Royal Soc. Edinburgh, 127A:595-614.

Potier-Ferry, M. [1981], The linearization principle for the stability of solutions of quasilinear parabolic equations. I. Arch. Rational Mech. Anal., 77:301-320.

Potier-Ferry, M. [1982], On the mathematical foundations of elastic stability theory. I. Arch. Rational Mech. Anal., 78:55-72.

Qi, Tang [1988], Almost-everywhere injectivity in nonlinear elasticity. Proc. Royal Soc. Edinburgh, 109A:79-95.

Qin, T. [1998], Symmetrizing the nonlinear elastodynamic system. J. Elasticity, 50:245-252.

Racke, R. and S. Zheng [1997], Global existence and asymptotic behavior in nonlinear thermoviscoelasticity. J. Differential Equations, 134:46-67.

Radin, C. [1987], Low temperature and the origin of crystalline symmetry. Internat. J. Modern Phys. B, 1:1157-1191.

Rybka, P. [1992], Dynamical modelling of phase transitions by means of viscoelasticity in many dimensions. Proc. Royal Soc. Edinburgh, 121A:101-138.

Serre, D. [2000], Systèmes de Lois de Conservation, Vols I,II. Diderot, Paris, 1996. English translation: Systems of Conservation Laws, Vols I,II, Cambridge Univ. Press, Cambridge.

Shu, Y. C. [2000], Heterogeneous thin films of martensitic materials. Arch. Ration. Mech. Anal., 153:39-90.

Sivaloganathan, J. [1986], Uniqueness of regular and singular equilibria for spherically symmetric problems of nonlinear elasticity. Arch. Rational Mech. Anal., 96:97-136.

Version Dec 16, $2001 \ldots \ldots$. Edited by wgm : Typeset on 26 January $2002-9$ h38 
Sivaloganathan, J. [1989], The generalised Hamilton-Jacobi inequality and the stability of equilibria in nonlinear elasticity. Arch. Rational Mech. Anal., 107:347-369.

Sivaloganathan, J. [1995], On the stability of cavitating equilibria. Quart. Appl. Math., 53:301-313.

Sivaloganathan, J. [1999], On cavitation and degenerate cavitation under internal hydrostatic pressure. Proc. R. Soc. Lond. Ser. A, 455:3645-3664.

Sivaloganathan, J. and S. J. Spector [2000a], On the optimal location of singularities arising in variational problems of nonlinear elasticity. J. Elasticity, 58:191-224.

Sivaloganathan, J. and S. J. Spector [2000b], On the existence of minimizers with prescribed singular points in nonlinear elasticity. J. Elasticity, 59:83-113. In recognition of the sixtieth birthday of Roger L. Fosdick (Blacksburg, VA, 1999).

Sivaloganathan, J. and S. J. Spector [2001], A construction of infinitely many singular weak solutions to the equations of nonlinear elasticity. Preprint.

Sobolevskii, P. E. [1966], Equations of parabolic type in Banach space. Amer. Math. Soc. Transl., 49:1-62.

Stoppelli, F. [1954], Un teorema di esistenza e di unicita relativo alle equazioni dell'elastostatica isoterma per deformazioni finite. Recherche Mat., 3:247-267.

Stoppelli, F. [1955], Sulla svilluppibilita in serie de potenze di un parametro delle soluzioni delle equazioni dell'elastostatica isoterma. Recherche Mat., 4:58-73, 1955.

Stringfellow, R. and R. Abeyaratne [1989], Cavitation in an elastomer - comparison of theory with experiment. Materials Science and Engineering A Structural Materials Properties, Microstructure and Processing, 112:127-131.

Stuart, C. A. [1985], Radially symmetric cavitation for hyperelastic materials. Ann. Inst. H. Poincaré. Anal. Non. Linéaire, 2:33-66.

Stuart, C. A. [1993], Estimating the critical radius for radially symmetric cavitation. Quart. Appl. Math., 51:251-263.

Šverák, V. [1988], Regularity properties of deformations with finite energy. Arch. Rational Mech. Anal., 100:105-127.

Šverák, V. [1991], Quasiconvex functions with subquadratic growth. Proc. Roy. Soc. Lond. A, 433:723-732.

Šverák, V. [1992], Rank-one convexity does not imply quasiconvexity. Proc. Royal Soc. Edinburgh, 120A:185-189.

Šverák, V. [1995], Lower-semicontinuity of variational integrals and compensated compactness. In Proc. International Congress of Mathematicians, Zurich 1994, Basel. Birkhaüser.

Šverák, V. and X. Yan [2000], A singular minimizer of a smooth strongly convex functional in three dimensions. Calc. Var. Partial Differential Equations, 10:213-221.

Sychev, M. A. [1999], A new approach to Young measure theory, relaxation and convergence in energy. Ann. Inst. H. Poincaré Anal. Non Linéaire, 16:773-812.

Version Dec 16, 2001 Edited by wgm : Typeset on 26 January $2002-9 \mathrm{~h} 38$ 
Sychev, M. A. [2001], Few remarks on differential inclusions. Preprint.

Sylvester, J. [1985], On the differentiability of $\mathrm{O}(n)$ invariant functions of symmetric matrices. Duke Math. J., 52:475-483.

Tadmor, E. B. , M. Ortiz and R. Phillips [1996], Quasicontinuum analysis of defects in solids. Phil. Mag. A, 73:1529-1563.

Taheri, A. [2001a], On Artin's braid group and polyconvexity in the calculus of variations. Preprint.

Taheri, A. [2001b], Quasiconvexity and uniqueness of stationary points in the multi-dimensional calculus of variations. Preprint.

Tartar, L. [1979], Compensated compactness and applications to partial differential equations. In R.J. Knops, editor, Nonlinear Analysis and Mechanics; Heriot-Watt Symposium, Vol. IV, pages 136-192. Pitman Research Notes in Mathematics.

Tartar, L. [1982], The compensated compactness method applied to systems of conservation laws. In Systems of Nonlinear Partial Differential Equations, J. M. Ball, editor, pages 263-285. NATO ASI Series, Vol. C111, Reidel.

Tartar, L. [1993], Some remarks on separately convex functions. In Proceedings of conference on Microstructures and phase transitions, IMA, Minneapolis, 1990.

Tonelli, L. [1921], Fondamenti di Calcolo delle Variazioni, Volumes I, II. Zanichelli, 1921-23.

Truesdell, C. and W. Noll [1965], The non-linear field theories of mechanics. In S. Flügge, editor, Handbuch der Physik, Berlin. Springer. Vol. III/3.

Valent, T. [1988], Boundary Value Problems of Finite Elasticity, volume 31 of Springer Tracts in Natural Philosophy. Springer-Verlag.

Vodop'yanov, S. K., V. M. Gol'dshtein and Yu. G. Reshetnyak [1979], The geometric properties of functions with generalized first derivatives. Russian Math. Surveys, 34:19-74.

Šilhavý, M. [1997], The Mechanics and Thermodynamics of Continuous Media. Springer.

Šilhavý, M. [2000], Differentiability properties of rotationally invariant functions. J. Elasticity, 58:225-232.

Wan, Y. H. and J. E. Marsden [1983], Symmetry and bifurcation in threedimensional elasticity, Part III: Stressed reference configurations. Arch. Rational Mech. Anal., 84:203-233.

Weiner, J. H. [1983], Statistical Mechanics of Elasticity. Wiley, New York.

Weinstein, A. [1985], A global invertibility theorem for manifolds with boundary. Proc. Royal Soc. Edinburgh, 99:283-284.

$\mathrm{Xu}$, C.-Y. [2000], Asymptotic Stability of Equilibria for Nonlinear Semiflows with Applications to Rotating Viscoelastic Rods. PhD thesis, Department of Mathematics, University of California, Berkeley, 2000.

Xu, C.-Y. and J. E. Marsden [1996], Asymptotic stability for equilibria of nonlinear semiflows with applications to rotating viscoelastic rods. I. Topol. Methods Nonlinear Anal., 7:271-297.

Version Dec 16, 2001 ....... Edited by wgm : Typeset on 26 January $2002-9$ h38 
Young, L. C. [1969], Lectures on the Calculus of Variations and Optimal Control Theory. Saunders, 1969. Reprinted by A.M.S. Chelsea.

Zhang, K. [1991], Energy minimizers in nonlinear elastostatics and the implicit function theorem. Arch. Rational Mech. Anal., 114:95-117.

Zhang, K. [2001], A two-well structure and intrinsic mountain pass points. Calc. Var. Partial Differential Equations, 13:231-264. 